\title{
Abnormal Gait Behavior Detection for Elderly Based on Enhanced Wigner-Ville Analysis and Cloud Incremental SVM Learning
}

\author{
Jian Luo, ${ }^{1,2}$ Jin Tang, ${ }^{1}$ and Xiaoming Xiao" \\ ${ }^{1}$ School of Information Science and Engineering, Central South University, Changsha, Hunan 410083, China \\ ${ }^{2}$ Hunan College of Information, Changsha, Hunan 410083, China \\ Correspondence should be addressed to Xiaoming Xiao; xmxiao@csu.edu.cn
}

Received 25 November 2015; Accepted 26 January 2016

Academic Editor: Parham Aarabi

Copyright (C) 2016 Jian Luo et al. This is an open access article distributed under the Creative Commons Attribution License, which permits unrestricted use, distribution, and reproduction in any medium, provided the original work is properly cited.

A cloud based health care system is proposed in this paper for the elderly by providing abnormal gait behavior detection, classification, online diagnosis, and remote aid service. Intelligent mobile terminals with triaxial acceleration sensor embedded are used to capture the movement and ambulation information of elderly. The collected signals are first enhanced by a Kalman filter. And the magnitude of signal vector features is then extracted and decomposed into a linear combination of enhanced Gabor atoms. The Wigner-Ville analysis method is introduced and the problem is studied by joint time-frequency analysis. In order to solve the large-scale abnormal behavior data lacking problem in training process, a cloud based incremental SVM (CI-SVM) learning method is proposed. The original abnormal behavior data are first used to get the initial SVM classifier. And the larger abnormal behavior data of elderly collected by mobile devices are then gathered in cloud platform to conduct incremental training and get the new SVM classifier. By the CI-SVM learning method, the knowledge of SVM classifier could be accumulated due to the dynamic incremental learning. Experimental results demonstrate that the proposed method is feasible and can be applied to aged care, emergency aid, and related fields.

\section{Introduction}

In most developed countries, the aging problem has existed for many decades, while, in developing countries, population aging has taken place relatively recently due to the demographic changes and strong gains in life expectancy. A UN study in 2013 said the number of older persons is 841 million in 2013 and the older population will almost triple by 2050, when it is expected to surpass the two billion mark. The share of the working-age population will continue to fall during the next four decades, to about 51 per cent in 2050 [1]. In contrast the old-age dependency ratio will grow rapidly, as a result a society's capacity for taking care of its elderly members could be overwhelmed.

Typically, most of the elderly people could prefer to live in their own homes rather than nursing homes especially in developing countries due to the high costs of living there.
However, the disability and health problems tend to grow rapidly. Elderly people who live alone with illness, health problems or action barrier, are especially in need of online supervision in case of emergency situations, such as sudden sign of illness, accidental fall, or shock. For example, the fall as one of "four giants of geriatrics" in old age, becomes a major public health problem, which could lead to serious injury even death. To prevent dangerous situations arising when elderly lives alone in their homes and to enhance the quality of their lives, more health care in general and more specialized services are required.

In this paper, an abnormal gait behavior detection system based on triaxial acceleration sensor and cloud incremental SVM Learning is proposed, for which automatic, ubiquitous health care can be realized and advanced technologies have been used to detect abnormal activities for elderly. In practice scenarios for elderly, the fall is the most important 
abnormal gait behaviors; in addition, syncope, stumbling, abnormal bend down, and spastic gait are typical abnormal gait behaviors. In traditional method, only fall is detected for alarm; that is to say when the system output alarms, the elderly might already fall down and get injured. If abnormal gait behavior (pseudo falls situations) in daily life could be collected, analyzed, and used for prediction of elderly health by remote, the old aged people could benefit from it, because the doctor from the Remote Telecare Center can detect changes in movement and recognize an underlying health problem for the elderly. For example, slipping against a wall ending with recovery (no fall) could be considered as neutral events and no alarm occurred in fall detection algorithms. The prevention of falls in the elderly is defined as "unintentionally coming to ground, or some lower level not as a consequence of sustaining a violent blow, loss of consciousness, sudden onset of paralysis as in stroke or an epileptic seizure" [2]. However, it is indeed an abnormal gait behavior; it should be detected and classified for further analysis by doctors.

In traditional methods, the fall detection algorithms are mainly focused on falling or not, and the algorithms are run on wearable devices with limited MCU or MPU inside. By lacking powerful remote communication function, the system only results in detection of a fall with sound, flash, or short messages for alarm. The detection of abnormal gait behavior of elderly people could be divided into two major categories. (1) Abnormal gait behavior is detected by video frames. In [3-9], gait silhouette features of human body or movement trajectory features are extracted from the surveillance videos. And they are used for abnormal gait behavior detection and analysis. Video based methods have some problems that are difficult to overcome, such as invasion of privacy, limited by the number, installation location, and capture view of cameras, which greatly limit their practical application. (2) Wearable devices are used for motion data collection and analysis. In [10-18] gravity acceleration sensors or gyrometers were explored for motion detection. In [10] the falling detection algorithm was introduced based on critical sensor thresholds by a wearable sensor system composed by a 3 -axis accelerometer and gyroscope. However, the proposed strategy of combining accelerometer and gyroscope measures using critical thresholds for the detection algorithm still required further study to result in a robust fall detection algorithm. In [11], the gait information of the elderly was captured using a motion-capture system, which consisted of at least six body-worn tags and wall-mounted sensors. And a machine learning classifier was proposed for recognition of health problems. However, the wall-mounted sensors made the system limited in indoor using. Li et al. [12] proposed a machine learning methods based on $K$-nearest neighbor algorithm to classify the activities from the data acquired from sensors based on sample data. However experiments are only done on small-scale movement data set (only six types of activities). Naranjo-Hernández et al. [13] explored a low-cost and wearable intelligent accelerometer sensor with a communication model based on IEEE 802.15.4 standard for the monitoring of human activities. However, the system was still weak in complex signal processing by only using an 8-bit PIC18F2431 microcontroller for data processing. In [14], torso and thigh wearable inertial sensors were used and the whole system was based on a body area network (BAN) for the comfort of the wearer during a long term application. But the experiments were still based on the data samples of young people's simulated activities, which were different in real-world practice. Bourke et al. in $[15,16]$ discussed a threshold-based algorithm to distinguish between Activities of Daily Living (ADL) and falls using a biaxial gyroscope sensor mounted on the trunk. However it achieved only a simple binary classification of falling or not falling. Ye et al. [17] presented a wireless sensor architecture based human falling detection system using 3-axis acceleration sensor to measure and collect the elderly people activities acceleration and transfer data by zigbee-3G network to remote medical monitoring platform. However, only one-class SVM classification algorithm together with a threshold to achieve judgment needed to be further improved in terms of original data classification. In [18] an algorithm for human activity-level detection was presented, based on the real-time processing of the signals produced by one wearable accelerometer. The algorithm was independent of the sensor orientation with respect to the body. However, the fixed thresholds using in the classifier for separating the classes might be sensitive in different scenarios and the appropriate use of the value should be taken into account in future research.

Estimating of daily activities using low-cost wearable devices is hard work especially when the actual data of elderly is limited. And there are no comprehensive solutions flexible enough for practical applications. The existing methods are usually poor in complex signal processing, accuracy classification, remote real-time application, and user experience due to the limitation of the wearable hardware that use simple algorithms. It may lead to an inaccurate estimation. And the wearable devices usually have big size that may be composed of several modules, including mobile power, sensors, control module, and communication module. As a result, it is usually not easy to wear and the elderly may be unwilling to wear all day. But with the popularization of smart phones, intelligent watches, and smart bracelets with high speed CPUs, wireless network, embedded acceleration sensors, or gyroscopes, it becomes easier for elderly to carry and benefit from them. Together with the powerful cloud platform, more complex algorithms can be introduced for abnormal behavior detection for elderly in their daily lives. In traditional methods, the abnormal behavior detections are most measured using the average acceleration or signal vector magnitude over a specific time period. The simple algorithms using the thresholds for activity recognition make it hard to distinguish complex schemes in daily life, such as climbing or descending stairs. And the threshold-based fall detection algorithms have difficulty in the choice of threshold values in real-world scenarios. If it sets too low, the negative events could be detected; if it sets too high, the positive events will not be detected. To address the above problems, the signal enhanced Wigner-Ville analysis method is proposed to extract both time and frequency features of abnormal gait behaviors. First, the Kalman filter is used for the acceleration signal enhancement using an autoregressive model. Second the strengthened 

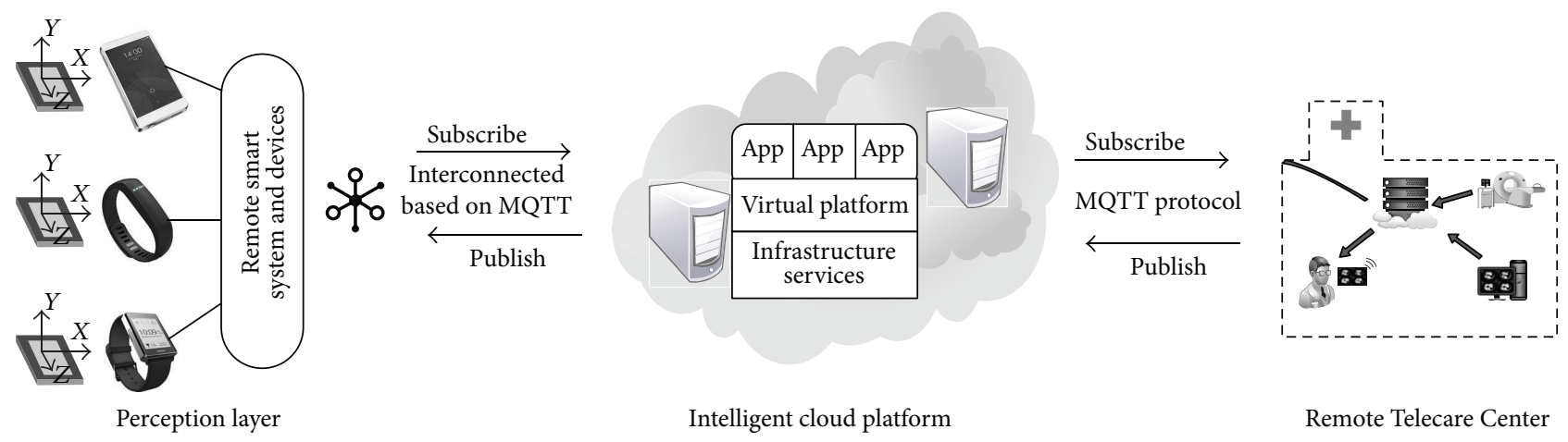

Remote Telecare Center

FIGURE 1: Architecture of abnormal gait behavior detection system based on intelligent cloud.

acceleration data are decomposed by the enhanced Gabor transform based on matching pursuit. Third, Wigner-Ville joint time-frequency analysis method is introduced to extract the discrete WVD (Wigner-Ville Distributions) energy matrices as time and frequency features for abnormal gait behavior. And the distribution of the enhanced joint time-frequency analysis features is for training and classification of abnormal gait behaviors. Time-frequency analysis that expands the signal in both time and frequency domain is widely used to study nonstationary time series. The technique for human gait classification based on the time-frequency analysis of radar signals received from human target in motion has already received good effect [19]. Time-frequency analysis provided easier extraction of the specific motion features and, compared to other methods, time-frequency distributions, which capture the instantaneous frequency laws that are most suitable for the underlying application.

However, there are still other problems that must be overcome in order to put the abnormal behavior detection system into practice. One of the most important difficulties is the lack of large-scale abnormal behavior data in training process. Usually, the limited healthy adult subjects with young ages were asked to imitate those abnormal behaviors. The collected imitated data set is then used in training replicating actual gait and falling of the elderly that make the recognition less accurate. Also the small-scale data set might make the existing methods less robust in actual applications by lack of relearning or incremental learning approaches. In order to overcome the problem, the cloud based incremental SVM (CI-SVM) learning method is introduced for abnormal behavior training and recognition. By using the incremental learning method based on cloud, the system can update the abnormal behavior data set through cloud platform and retrain the classifier using the incremental data set. As a result, the knowledge is accumulated which makes the online abnormal behavior recognition more robust.

The contributions of this paper are the following: (1) by utilizing the intelligent cloud platform, a novel cloud based abnormal behavior detection system for elderly health care is introduced. The purpose of the system is to ensure longer and generally healthier lives of individuals for old aged people; (2) by using the strengthen Kalman filter, enhanced Gabor atoms decomposition, and Wigner-Ville analysis method, a novel abnormal gait behavior feature extracting method is proposed. Both time and frequency features of abnormal gait are extracted which makes the system have high reliability and capacity of resisting disturbance; (3) by introducing the incremental learning method into the health care system, a cloud based incremental SVM learning algorithm is explored which helps to construct the more accuracy classifier for abnormal behavior detection.

The rest of this paper is organized as follows. Section 2 presents the design of cloud based abnormal behavior detection system. Section 3 presents a novel abnormal gait behavior feature extracting method. Section 4 presents the incremental SVM learning algorithm. Section 5 presents experimental results and Section 6 concludes the paper.

\section{Design of Cloud Based Abnormal Behavior Detection System}

2.1. Overview. In the proposed diagram (see Figure 1), the architecture of abnormal gait behavior detection system is illustrated. The system is composed of perception layer, intelligent cloud platform, and Remote Telecare Center. The remote smart system and devices (smart phones, intelligent watches, and smart bracelets) with sensors embedded are in the perception layer. It connects the intelligent cloud platform by wireless or mobile networks using MQTT protocol. The triaxial acceleration sensors embedded in the smart terminals are used to measure the body motion data, including $X Y Z$ three-axis acceleration data. The acceleration data are processed in the smart device first with some simple algorithms, such as finite impulse response filter, prejudgment. If there is any unusual data collected, it would be published to the intelligent cloud platform for further analysis through MQTT protocol. The intelligent cloud platform integrates remote smart devices and runs Web-based applications, such as key application for abnormal behavior incremental training and online recognition, other applications for data encoding, message push and user register, and management. The Remote Telecare Center can be designed as the health care center for elderly distributed in different locations. The doctors from the center can get the motion information 


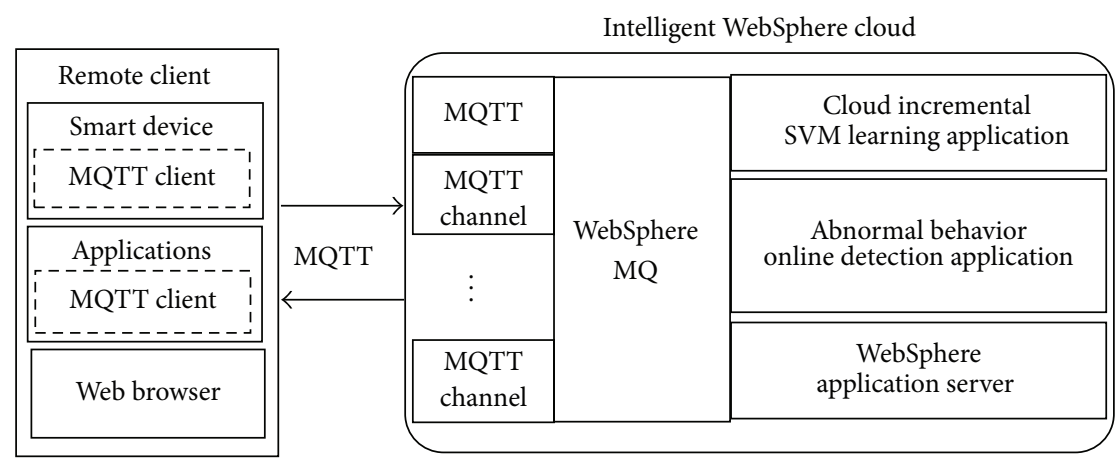

FIGURE 2: Architecture of intelligent WebSphere cloud platform.

of elderly in daily life for remote monitoring. If there are unusual gait events that occur in a period, the doctor can make a remote diagnosis and ask the old aged people for further checking with their more complex pathologies. The emergency rescue information can also be received by the Remote Telecare Center for confirmation and processing if elderly fall and get injured in their own homes. Doctors in the RTC can also be able to check patients' status at any time.

2.2. Intelligent WebSphere Cloud Platform for Online Learning and Recognition. WebSphere MQ Telemetry and WebSphere Application Server of IBM are introduced to construct the cloud platform for cloud computing and message pushing. WebSphere MQ Telemetry Transport (MQTT) is a "light weight" messaging protocol developed by IBM. MQTT is optimized for the implementation on low-cost, battery-operated devices with limited processing, and storage resources such as wireless sensor devices [20]. WebSphere MQ enables programs to communicate with one another across a network of unlike components (processors, operating systems, subsystems, and communication protocols) using a consistent application programming interface. It provides real-time access to a range of mobile devices, remote sensors, actuators, and other telemetry devices leveraging WebSphere MQ. As a result, it is widely used in connectivity for internet of things and mobile devices.

MQTT is based on publish-subscribe. By using push technology, clients from different platforms can subscribe the interested information in different channels published in cloud servicer. Once the subscription information is updated, such as a new email that arrived, the message will push to the specific clients that have subscribed to the topics. In the same time, clients can publish the message by MQTT Broker to one specific receiver or multiple receivers.

MQTT protocol is composed of header and payload. The min size of the fix header is only 2 bytes. The payload can be defined by user to transmit the messages that are agreed on before. In this paper, MQTT is used to transform message from remote smart devices to Remote Telecare Center with the payload containing the detailed message.

WebSphere application server (WAS) is a software framework that performs the role of a Web application server.
WebSphere MQ works as middleware to connect the remote client and virtual applications in WAS. Data exchange and integration are achieved by MQTT protocol. The virtual abnormal behavior detection application with flexible detection algorithms is designed and deployed on the WAS. By using the cloud computing technology, more complex and time-consuming algorithms can be introduced than in remote smart devices. The remotely located smart devices collect real-time data of abnormal behaviors with simple algorithms for preprocessing. The abnormal data is then sent to the cloud platform for further analysis and the results will be sent to the Remote Telecare Center by push technology. The architecture of intelligent WebSphere cloud platform is shown in Figure 2.

From Figure 2, it is seen that the remote smart devices with acceleration sensors embedded are in perception layer. The collected data and information are published or subscribed by embedded app through MQTT. The message queues form different MQTT channels are well dispatched and managed by WebSphere MQ Telemetry server. The abnormal behavior training and detection processes are achieved online by the virtual applications deployed in WebSphere Application Server. The Remote Telecare Center accesses information of elderly located in different remote area by MQTT clients, mobile device, or Web browser through publish-subscribe.

All clients from smart terminals, elderly users, Remote Telecare Centers (RTC), or monitoring-centers must register in the cloud server and get the unique identification as Token. Clients from RTC will subscribe to the topics from elderly that need attention and two-way data binding is achieved between elderly users and professor servicers.

In daily life, once an abnormal behavior is detected by the registered smart mobile device, for example, falling down or blackouts, the message will be sent to the cloud servicer. After online recognition by virtual detection applications, the message with detailed information, for example, name of user, home address, phone number, and location, will be pushed to all the clients that have subscribed to the topics including family members. The RTC or other clients could make a voice call to ensure the message is sent. If the emergency event occurs, immediate actions will be taken to ensure the life and health safety of the elderly. 


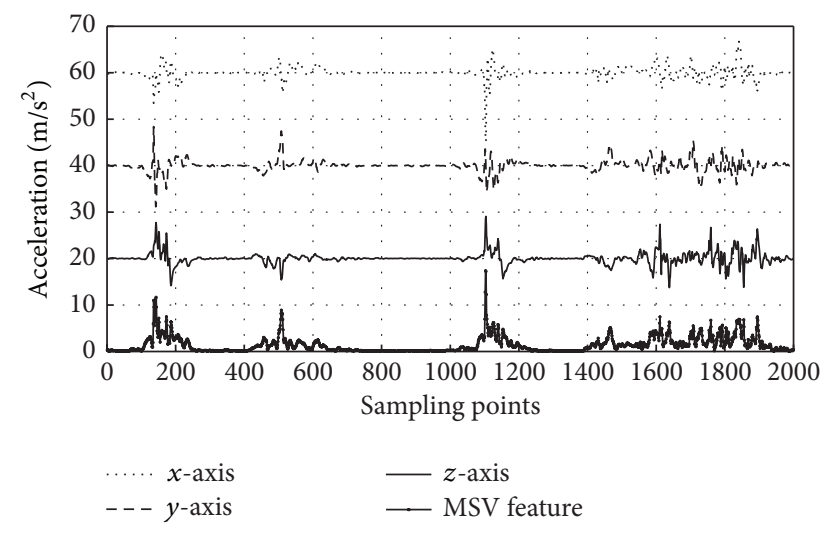

Figure 3: Data sequence of the acceleration and MSV features.

The virtual behavior detection application is run background at the WebSphere cloud platform. An uploaded abnormal three-axis motion data is checked in real-time. When a message with abnormal behavior information is received, the effective data is then extracted from the data packet. The useful data is then analyzed by an abnormal behavior detection algorithm.

\section{Enhanced Wigner-Ville Analysis of Three-Dimensional Human Body Motion Information}

3.1. 3D Motion Data Capturing and Enhancing. In this paper, a smart hand phone with accelerometer embedded is used to collect the motion data. The hand phone is put into the pocket of garment. A priori knowledge about sensor's correct orientation is not necessary by using of signal vector magnitude as motion features. The Cartesian coordinate system $X Y Z$ is defined first as shown in Figure 1. The triaxial acceleration sensors embedded in the smart terminals can collect body motion data in $X, Y$, and $Z$ axes separately. The acceleration data may differ largely due to the different motion behaviors, such as walking, falling, climbing stairs, and sitting down. Figure 3 shows the stored acceleration data in three axes over a time period.

Let $X_{t}, Y_{t}$, and $Z_{t}$ denote the function for acceleration data of three axes separately. In order to reduce the error caused by the initial location of wearing acceleration sensor, the magnitude of signal vector (MSV) is defined as $\operatorname{MSV}(t)=\sqrt{\left(X_{t}-X_{\text {offset }}\right)^{2}+\left(Y_{t}-Y_{\text {offset }}\right)^{2}+\left(Z_{t}-Z_{\text {offset }}\right)^{2}}$, where $X_{\text {offset }}, Y_{\text {offset }}$, and $Z_{\text {offset }}$ denote the acceleration bias values.

Before MSV feature extraction, the acceleration signal is enhanced using a masking threshold constrained Kalman filter discussed in [21]. The Kalman filter [22] is well known applied tool and effective technique in the statistical signal processing field, which has found numerous applications in fields related to control and estimation of dynamic systems.
In using of the Kalman filter, we modeled the $n$th sample of clean acceleration signal $s(n)$ as $p$ th order quasistationary $\mathrm{AR}$ (autoregressive) process as shown in

$$
s(n)=\sum_{i=1}^{p} a_{i} s(n-i)+u(n),
$$

where $a_{i}$ is the $i$ th AR coefficient and $u(n)$ is the residual signal assuming as a Gaussian white noise with variance $\sigma_{u}^{2}$. The observed signal $y(n)$ is assumed to be contained by a zeromean additive Gaussian noise $v(n)$ with variance $\sigma_{v}^{2}$. And the noisy model is denoted by

$$
y(n)=s(n)+v(n),
$$

where $v(n)$ is the background noise process. Using a vector Kalman filter, the state-space model of the measured acceleration signal is expressed as [21]

$$
\begin{aligned}
& x(n)=F x(n-1)+G u(n), \\
& y(n)=H x(n)+v(n),
\end{aligned}
$$

where $x(n)=[s(n-p+1) \cdots s(n)]^{T}, y(n)=[y(n-p+$ 1) $\cdots y(n)]^{T}, v(n)=[v(n-p+1) \cdots v(n)]^{T}$. Consider the following:

$$
\begin{gathered}
F=\left[\begin{array}{ccccc}
0 & 1 & 0 & \cdots & 0 \\
0 & 0 & 1 & \cdots & 0 \\
\vdots & \vdots & \vdots & \ddots & \vdots \\
0 & 0 & 0 & \cdots & 1 \\
a_{p} & a_{p-1} & a_{p-2} & \cdots & a_{1}
\end{array}\right]_{p \times p}, \\
H^{T}=G=\left[\begin{array}{c}
0 \\
0 \\
\vdots \\
1
\end{array}\right]_{p \times 1}
\end{gathered}
$$

If parameters $a=\left[\begin{array}{lllll}a_{p} & a_{p-1} & a_{p-2} & \cdots & a_{1}\end{array}\right], \sigma_{u}^{2}$, and $\sigma_{v}^{2}$ are known, the optimal estimate of $s(n)$ can be obtained from the Kalman filter. The $\widehat{x}(n \mid n)$ represents the filtered estimate of the state vector $x(n)$ and is calculated by the method proposed in [21]:

$$
\begin{aligned}
e(n) & =y(n)-\hat{x}(n \mid n-1)-\bar{v}, \\
K(n) & =P(n \mid n-1) \times(P(n \mid n-1)+R)^{-1}, \\
\hat{x}(n \mid n) & =\widehat{x}(n \mid n-1)+K(n) \times e(n), \\
P(n \mid n) & =(I-K(n) \times P(n \mid n-1)), \\
\widehat{x}(n+1 \mid n) & =F \hat{x}(n \mid n)+G \bar{u}, \\
P(n+1 \mid n) & =F P(n \mid n) F^{T}+G G^{T} \sigma_{u}^{2},
\end{aligned}
$$

where $e(n)$ is the innovation vector; $\bar{v}$ is the mean of $v(n) ; K(n)$ is the Kalman gain; $\widehat{x}(n \mid n-1)$ is the minimum mean-square estimate of the state vector $x(n)$ given the past observations; 

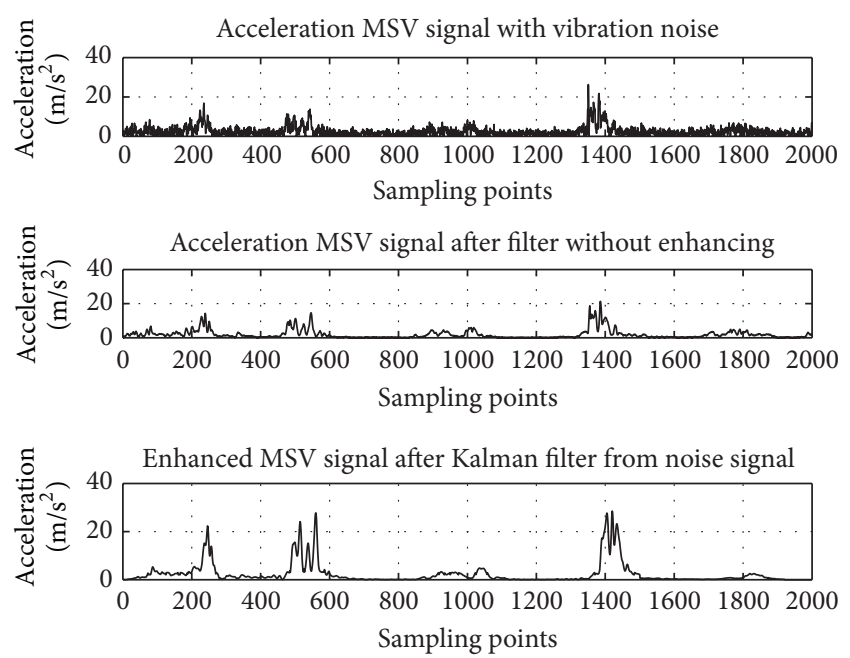

FIGURE 4: Comparison of MSV signals before and after enhancement by Kalman filter.

$P(n \mid n)$ is the filtered state error covariance matrix; and $P(n \mid n-1)$ is the predicted state error covariance matrix. The value of the enhanced acceleration signal $\widehat{s}(n)$ is the last value in the vector $\widehat{x}(n \mid n)$. In our acceleration collecting system, the sampling frequency is set to $100 \mathrm{~Hz}$; the frame size of the motion signal was 256 sample points, that is, $2.56 \mathrm{~s} /$ frame. The AR order for estimation is set to be 32 , and the coefficients are updated every frame. Two times the data are used to predict the AR parameters that include the previous enhanced frame and the current noisy embedded frame.

Figure 4 shows the comparison of MSV signal before and after enhancement by Kalman filter. From the illustration, it can be seen that the vibration noise created by vibration generator has been reduced greatly and the quality of the acceleration data is increased after enhancement by Kalman filter based on AR model. The acceleration data enhancement algorithm makes the system valid for practical scenarios.

\subsection{Enhanced Gabor Atom Feature Extracting Based on MP.} Matching pursuit (MP) is a suboptimal, nonlinear, iterative procedure, decomposing a signal into a linear sum of known functions (atoms) which are chosen from a redundant set (dictionary) [23]. The decomposition process of MP is achieved by iteration. The iteration number is determined by the given residual. In the first step, an initial atom $g_{\gamma}$ is chosen from the dictionary $(D)$ that has the largest product with the signal. The residual between the given signal and the selected atom is calculated. And it is used as new given signal to be decomposed. In successive iterations, atoms that have the largest products with the new given signal are chosen. Repeating the procedure until the signal is decomposed with the given accuracy, the procedure is defined as

$$
\begin{aligned}
R^{0} x & =x, \\
R^{m} x & =\left\langle R^{m} x, g_{\gamma_{m}}\right\rangle g_{\gamma_{m}}+R^{m+1} x, \\
g_{\gamma_{m}} & =\underset{g_{\gamma_{i}} \in D}{\operatorname{argmax}}\left|\left\langle R^{m} x, g_{\gamma_{i}}\right\rangle\right|,
\end{aligned}
$$

where $x$ is the signal to be composed and $\langle\cdot\rangle$ is operation for inner product. The atom fitted to the signal $x$ in the $m$ th iteration of MP as $g_{\gamma_{m}}$ and $m$ th residual as $R^{m} x$. The expansion result is equal to

$$
x \approx \sum_{m=0}^{M^{\prime}-1}\left\langle R^{m} x, g_{\gamma_{m}}\right\rangle g_{\gamma_{m}},
$$

where $M^{\prime}$ denotes the number of iterations. The dictionary $D$ is usually constructed from Gabor functions. As given in (8) the Gabor atom function is denoted by

$$
g_{\gamma}=K(\gamma) e^{-\pi((t-u) / s)} \cos (v t+\phi),
$$

where $\gamma=(u, v, s, \phi)$ denotes parameters of time-frequency. They are equal factors of shift, frequency, scale, and phase, respectively. And $K(\gamma)$ is such that $\left\|g_{\gamma}\right\|=1$. Using Gabor atoms, the signal can be represented associated with the timefrequency distributions. However, the frequency of the Gabor atom does not change over time. As acceleration signals are typical nonstationary signals, wherein the vibration occurs on a time-frequency area, lots of atoms should be selected to approximate an input signal by MP method to decompose the vibration. In order to solve the problems, an enhanced Gabor function is introduced to construct the dictionary that is denoted by [24]

$$
g_{\gamma}=K(\gamma) e^{-\pi((t-u) / s)} \cos \left(w(t-u)+\frac{c}{2}(t-u)^{2}\right),
$$

where $s, u, w, c$ are denoted as scale, time shift, frequency, and phase modulation rate separately. The vibration problems can be overcome by the introduction of the phase modulation rate $c$. The dictionary $D$ is then composed of enhanced Gabor functions for a time-frequency analysis of acceleration signals.

Parameters $\gamma=(s, u, w, c)$ in functions $g_{\gamma}$ form a 4dimensional continuous subspace of $R^{4}$ which can be defined as the infinite set $D_{\infty}$. The complete enhanced Gabor dictionary $D$ can be constructed by subsampling $D_{\infty}$ proposed in [24] with parameters:

$$
\begin{aligned}
\gamma & =(s, u, w, c) \\
& =\left\{\left(a^{j}, n a^{j} \Delta u, k a^{-j} \Delta w, l a^{-2 j} \Delta c\right), j, n, k, l \in \mathbb{Z}\right\} .
\end{aligned}
$$

3.3. Wigner-Ville Time-Frequency Analysis. From (7), a timefrequency distribution of the signal's energy can be gained, by summing WVD (Wigner-Ville distributions) of selected $W$ functions and explicitly omitting cross-terms [25]:

$$
E x(t, f)=\sum_{m=0}^{M^{\prime}}\left|\left\langle R^{m} x, g_{\gamma_{m}}\right\rangle\right|^{2} W g_{\gamma_{m}}(t, f) .
$$

The discrete WVD energy matrices with time-frequency distribution characteristic are then extracted as features to achieve classification and recognition of abnormal behavior.

Let $\operatorname{MSV}_{\text {Kalman }}(n), n=1,2, \ldots, N$, denote the signal vector magnitude of triaxial acceleration after signal 
enhancement by Kalman filter. $N$ equals the max number of sampling points in an action cycle. $\operatorname{MSV}_{\text {Kalman }}(n)$ is then decomposed into $M^{\prime}$ linear combination of enhanced Gabor atoms by the method of MP. The Wigner-Ville time-frequency analysis method is then introduced to extract the discrete WVD energy matrices which are denoted by

$$
D_{1: N}^{i}=\left(\mathrm{VWD}_{1}^{i}, \ldots, \mathrm{VWD}_{n}^{i}, \ldots, \mathrm{VWD}_{N}^{i}\right) \in R^{M \times N}
$$

where $i=1,2, \ldots, I, \mathrm{VWD}_{n}^{i}$ equals the discrete WVD energy matrix of the $i$ th training sample at timing $n$.

\section{Classification and Recognition Based on CI-SVM}

4.1. Support Vector Machine. Support vector machine (SVM) introduced by Vapnik [26] has been proposed as a very effective tool for regression estimation, pattern classification, and machine learning that have gained outstanding generalization in the last decade. For a classification problem, the use of SVM is to find the maximum margin hyperplane which separates the labeled training samples. The hyperplane can be described as

$$
\omega^{T} x+b=0, \quad x \in R^{n}
$$

Given training data $\left\{x_{i}, y_{i}\right\}, i=1,2,3, \ldots, n, x_{i} \in R^{m}$, $y=1$ or -1 , the hyperplane can be found by solving a convex quadratic programming problem [27]:

$$
L(\omega, b, \alpha)=\frac{1}{2} w^{T} w-\sum_{i=1}^{n} \alpha_{i}\left[y_{i}\left(w^{T} x_{i}+b\right)-1\right]
$$

s.t. $\quad y_{i}\left(w^{T} x+b\right) \geq 1, i=1,2, \ldots, n$.

The equation then can be substituted into its dual form:

$$
\begin{array}{ll}
\max & L(\omega, b, \alpha)=\sum_{i=1}^{n} \alpha_{i}-\frac{1}{2} \sum_{i=1}^{n} \sum_{j=1}^{n} \alpha_{i} \alpha_{j} y_{i} y_{j} K\left(x_{i} \cdot x_{j}\right) \\
\text { s.t. } & \sum_{i=1}^{n} \alpha_{i} y_{i}=0 \\
& 0 \leq \alpha \leq C, \quad i=1,2, \ldots, n,
\end{array}
$$

where $\alpha=\left(\alpha_{1}, \ldots, \alpha_{n}\right)$ denotes Lagrangian multiplier and $C$ is a constant that is used to control the amount of regularization. $K(\cdot)$ is kernel function that can be extended to nonlinear case by transforming the input nonlinearly into a highdimension feature space. The examples whose Lagrangian multiplier is $\alpha_{i} \neq 0$ are called support vectors (SVs). By solving the optimal problem in (15) the corresponding SVM classification function is denoted by

$$
f(x)=\operatorname{sign}\left[\sum_{i=1}^{n} \alpha_{i} y_{y} K\left(x \cdot x_{i}\right)+b\right] .
$$

To get an optimal solution $\alpha=\left(\alpha_{1}, \ldots, \alpha_{n}\right)$, the following Karushe Kuhne Tucker (KKT) conditions must be satisfied for every sample $x_{i}$ [28]:

$$
\begin{aligned}
& \alpha_{i}=0 \Longrightarrow f\left(x_{i}\right)>1 \\
& \quad \text { or } f\left(x_{i}\right)<-1 \\
& 0<\alpha_{i}<C \Longrightarrow f\left(x_{i}\right)=1 \\
& \quad \text { or } f\left(x_{i}\right)=-1 \\
& \alpha_{i}=C \Longrightarrow-1<f\left(x_{i}\right)<1 .
\end{aligned}
$$

For a training sample $x_{i}$, if $\alpha_{i}=0$, then it lies outside the boundaries of the separating margin; if $0<\alpha_{i}<C$, then it lies on either of the boundaries; and if $\alpha_{i}=C$, then it lies inside the boundaries of the separating margin. $f(x)= \pm 1$ are the boundaries of the separating margin.

4.2. Cloud Incremental SVM (CI-SVM) Learning. In abnormal behavior detection or falling detection process, for safety reasons [10-18], the training data set is usually based on an attempt by young people to imitate those behaviors. Assumption of imitated gait in young volunteers replicating actual gait and falling of the elderly can make the recognition result less accurate in real applications. It is usually hard to quantify the accuracy of imitated gate and actual data from the elderly due to the lack of large-scale actual abnormal behavior data. And no paper so far has discussed this problem. In traditional abnormal behavior detection methods, the training data are fixed and most of them are imitated data. When a new incremental observation arrives, learning has to begin from search with the whole training data set. It might greatly influence its applications.

In order to overcome these problems, the cloud based Incremental SVM learning method is introduced which is shown as Figure 5. The original imitated abnormal behavior data are first used to train the SVM for classification online. And the actual abnormal behavior data of elderly collected by the mobile smart devices with wireless network are then gathered in cloud platform to form the incremental training data set. The incremental training data are then used as new and actual observation data to update the last SVM classification parameters. In the same time the useless samples are discarded using an elimination algorithm based on safe distance and the counter sifting to keep the testing accuracy and reduced the training time. By the proposed incremental cloud-SVM learning method, the SVM classifier could be more accurate in actual application due to the dynamic incremental learning.

Traditionally, the training of support vector machines is a difficult issue in classifying large-scale data set since it requires solving a quadratic programming $(\mathrm{QP})$ problem in a number of coefficients equal to the number of training examples. Another difficulty is that almost all SVM algorithms at hand are not applicable online, that is, in cases where data are sequentially obtained and learning has to be done from the first data [29]. The incremental learning method, as one of the key technologies of intelligence knowledge discovering, has 


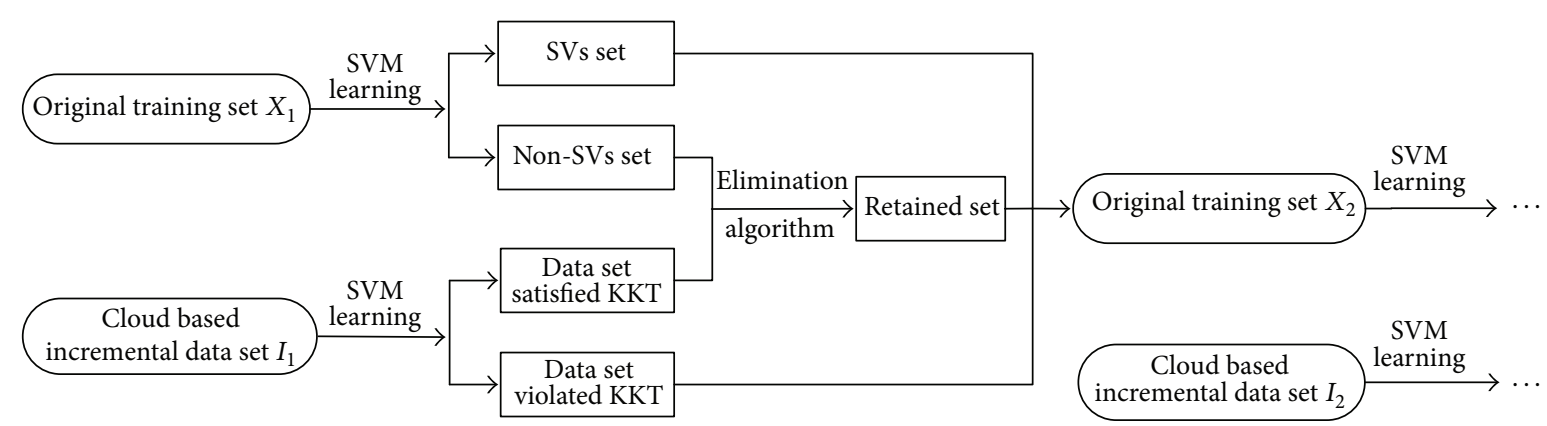

Figure 5: Process of CI-SVM learning.

then developed and been introduced into the SVM learning over large-scale date sets. It opens a new way to overcome the existing shortage and difficulties.

In paper [30], it first analyzed the properties of SVs set thoroughly and introduced a new learning method, which extends the SVM classification algorithm to incremental learning area. However, in the proposed method only the support vectors and highly related data were preserved for the next increment, and all other data samples were discarded which might carry useful information for classification. As a result the accuracy might be affected in the following increments process.

In order to overcome the problem, an elimination algorithm based on safe distance and the counter sifting is introduced. It is obviously that the samples closed to the boundaries of the separating margins have high probability to become the SVs in the next incremental training process. So the safe distance $d_{\text {safe }}$ are defined to separate the training samples into two set $X_{\text {safe }}$ and $X_{\text {latent }}$, where $X_{\text {latent }}$ denotes the data set containing important information and $X_{\text {safe }}$ describes the data samples of less important. Let $d_{i}$ denote the distance between $x_{i}$ samples in the training set and the separating margin plane. If $d_{i}>d_{\text {safe }}, x_{i}$ belongs to data set $X_{\text {safe }}$; otherwise it belongs to $X_{\text {latent }}$. The samples in $X_{\text {safe }}$ could be deleted due to less usefulness. However only using the safe distance to determine the removing data could be less reliable. The threshold of the safe distance might influence the accuracy directly. In order to make the elimination algorithm more effective and robust, the counter sifting is introduced. In the incremental SVM training process, it is found that if the samples satisfy the KKT conditions time and again it could have high probability to satisfy the KKT conditions next time. Otherwise, the samples of SVs could violate the KKT conditions over and over again. Based on the above knowledge, the samples with high probability to satisfy the KKT conditions could be ignored, which may be less important for SVM training. The counters $\mathrm{Cn}_{i}$ and a threshold $r$ are then set for all of the samples. In each incremental SVM training process, if sample $x_{i}$ satisfies the KKT conditions, set $c n_{i}=c n_{i}+1$; otherwise clear $c n_{i}$ to zero. When $c n_{i}>r$, the $x_{i}$ sample is marked as candidate to be deleted. If $x_{i}$ sample belongs to the data set $X_{\text {safe }}$ at the same time, $x_{i}$ could be removed finally. The detail algorithm of CI-SVM is described and symbols used in algorithm of CI-SVM will be as follows:

$\Omega_{o}^{k}$ : SVM classifier learned using the $k$ th original training data set;

$X_{o}^{k}$ : the $k$ th original data set for training;

$X_{I}^{s_{k}}$ : the data set from the newly incremental data samples satisfied the KKT conditions of $\Omega_{o}^{k}$;

$X_{o}^{s v_{k}}$ : SV data set of $\Omega_{o}^{k}$;

$X_{I}^{k}$ : the $k$ th original data set $(k=0$ denotes the original training data set);

$X_{o}^{n s v_{k}}$ : non-SV data set of $\Omega_{o}^{k}$;

$X_{I}^{v_{k}}$ : the data set from the newly incremental data sample which violate the KKT conditions of $\Omega_{o}^{k}$;

$X_{u}^{s_{k}}$ : union of $X_{o}^{n s v_{k}}$ and $X_{I}^{s_{k}}$;

$X_{r}^{s_{k}}$ : the rest data set of $X_{u}^{s_{k}}$ after elimination algorithm.

\section{The Algorithm of CI-SVM Is Summarized and Stated as Follows}

(1) Read the training data set. If the data set is null then end the algorithm else go to step (2).

(2) Obtain the newly incremental data set $X_{I}^{k}$ from the training data set. If $k=0$, let the original training data set $X_{o}^{0}=X_{I}^{0}$, get the original SVM classifier $\Omega_{o}^{0}$ by training on $X_{o}^{0}$, and set $k=k+1$ and go to step (1); if $k \neq 0$, go to step (3).

(3) If no samples from $X_{I}^{k}$ violate the KKT conditions, go to step (1), else divide the data set $X_{I}^{k}$ into $X_{I}^{s_{k}}$ and $X_{I}^{v_{k}}$ according the check results.

(4) Combine data set $X_{o}^{n s v_{k}}$ and $X_{I}^{s_{k}}$ to form the union set $X_{u}^{s_{k}}$ and calculate the remaining data set of $X_{r}^{s_{k}}$ by elimination algorithm on $X_{u}^{s_{k}}$.

(5) Update $X_{o}^{k+1}$ by combining the data set $X_{r}^{s_{k}}, X_{o}^{s v_{k}}$, and $X_{I}^{v_{k}}$ together and get the newly original SVM classifier 


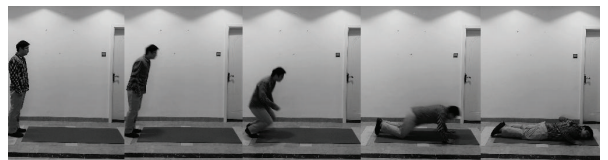

(a)

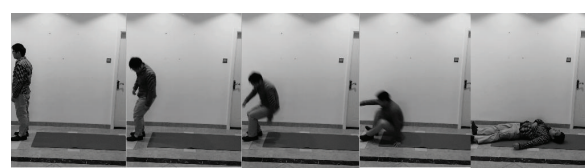

(b)

Figure 6: Demo video frames illustration shown to the study participants. (a) Forward falling. (b) Backward falling.

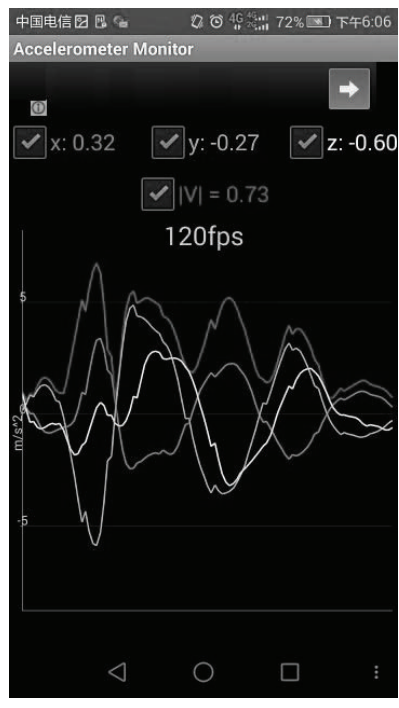

(a)

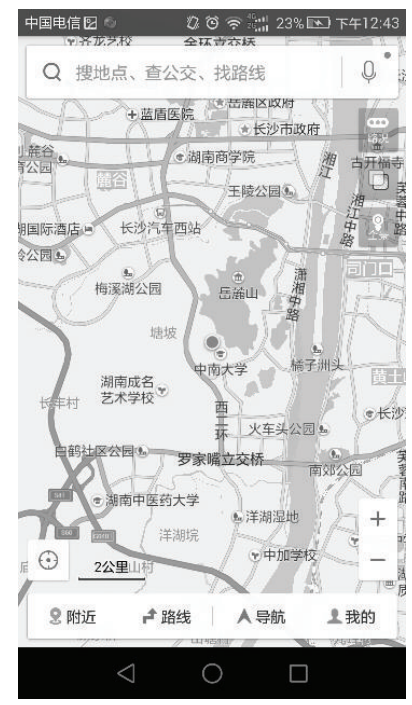

(b)

FIGURE 7: (a) Screenshot of the acceleration recording GUI on the smart phone. (b) GPS-assisted geolocalization outdoor.

$\Omega_{o}^{k+1}$ by training on $X_{o}^{k+1}$. Calculate SV data set $X_{o}^{s v_{k+1}}$ and none of the SV data set $X_{o}^{n s v_{k+1}}$; set $k=k+1$ and go to step (1).

\section{Experiment Results}

In order to evaluate the effectiveness of our proposed algorithm, experiments were carried out. First, twenty 20-35 years old people (10 men and 10 women) were asked to imitate the daily behaviors, that is, walking, sitting, standing, squatting, and falling. And additional five 45 years and five 55 years old people were chosen to execute the same abnormal behaviors for incremental learning reasons. The participants were required to perform the given different abnormal behavior actions and each behavior is recorded 10 times for every object. The sample data were collected at the sampling frequency of $100 \mathrm{~Hz}$. As a result, 2400 imitated sample data were collected from young people and they were divided into two groups. One group was used for training and the other group for recognition by our proposed algorithm. Another 1200 actual sample data of old people were collected to form 4 group data sets. They included two incremental data sets $I_{1}$ and $I_{2}$ with 300 data samples for each set, and two recognition data sets with another 300 data samples for each set according to their ages. The original 1200 imitated training data samples were first used to train the SVM classifier denoted by $\Omega_{o}^{0}$. And the remaining data sets were used for recognition. Then the $I_{1}$ set with 300 incremental data samples of 45-year-olds was used to conduct first time cloud based incremental SVM learning; the SVM classifier is updated and denoted by $\Omega_{o}^{1}$. The remaining data samples were used for recognition. In the last step, the $I_{2}$ set with 300 incremental data samples of 50-year-olds was used for the second time incremental learning and the classifier is updated to $\Omega_{o}^{2}$. By testing the rest of the samples, the recognition rates are obtained. Figure 6 shows the demo video frames where participants imitate the daily abnormal behaviors as well as some other daily life activities.

The experiment is implemented in a Huawei C199 smart phone with the following specification:

(1) CPU: Snapdragon MSM8928, Quad-core, $1.6 \mathrm{GHz}$.

(2) RAM: 2 GB.

(3) ROM: 16 GB.

(4) OS version: Android OS 4.4.2.

Figure 7 shows the screenshot of the acceleration recording GUI on the smart phone and GPS-assisted geolocalization outdoor. When the abnormal data collected by remote smart terminal is pushed to the virtual detection application running on the cloud platform, the preprocessing is conducted. And then MP decomposition process is introduced. Figure 8 shows the MP decomposition process of falling down in different iteration times. And Table 1 illustrates the parameters 

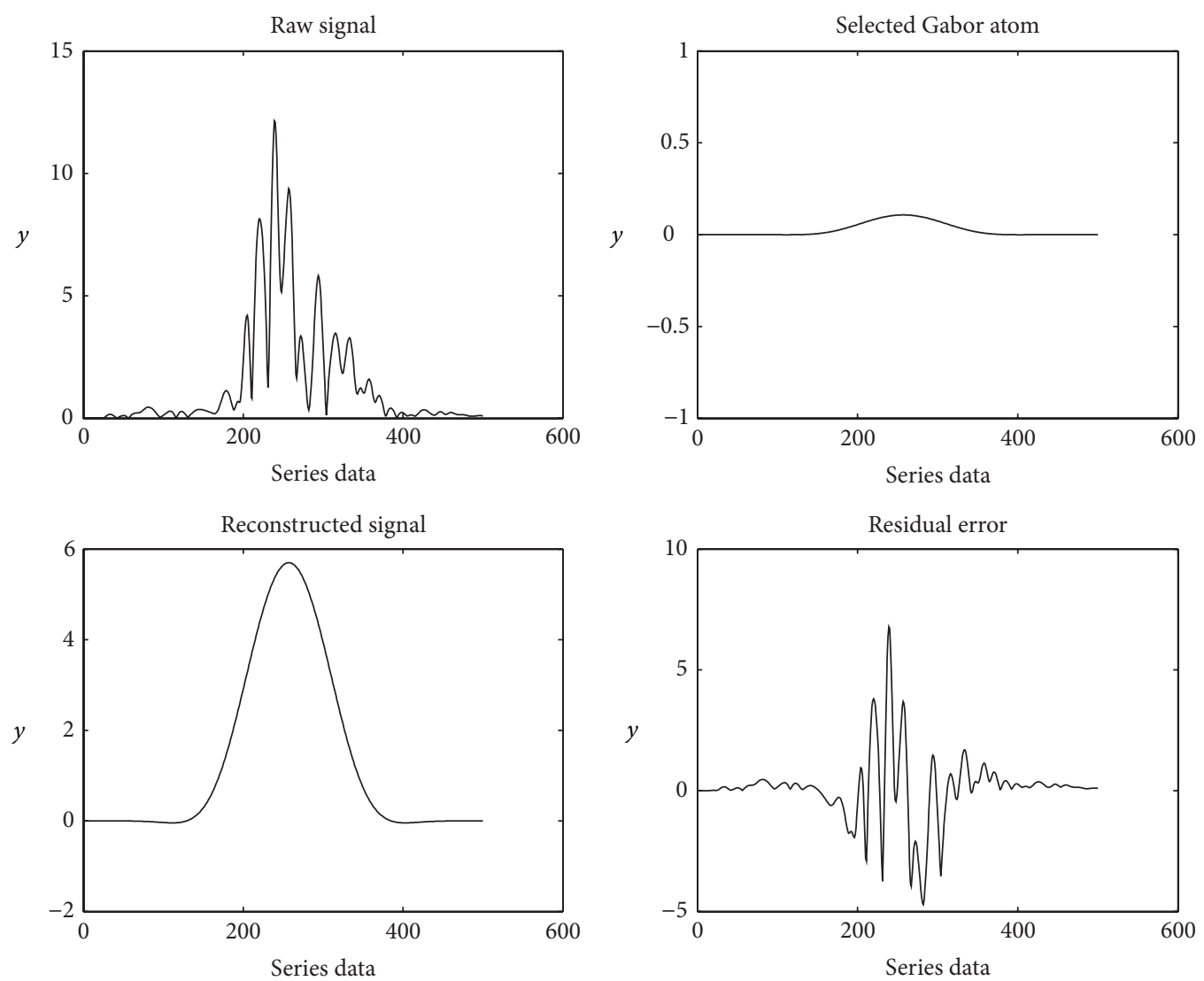

(a)
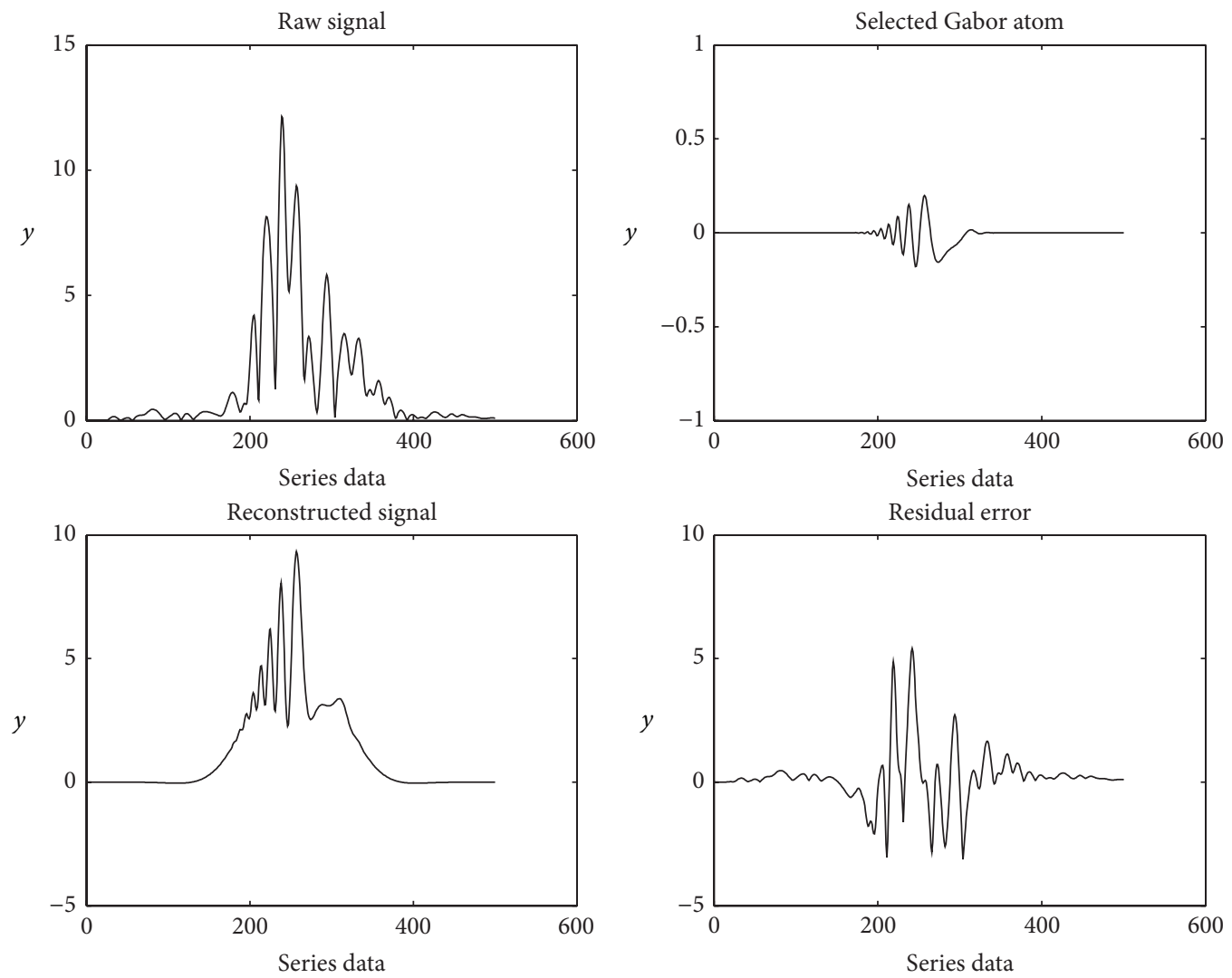

(b)

FIgUre 8: Continued. 

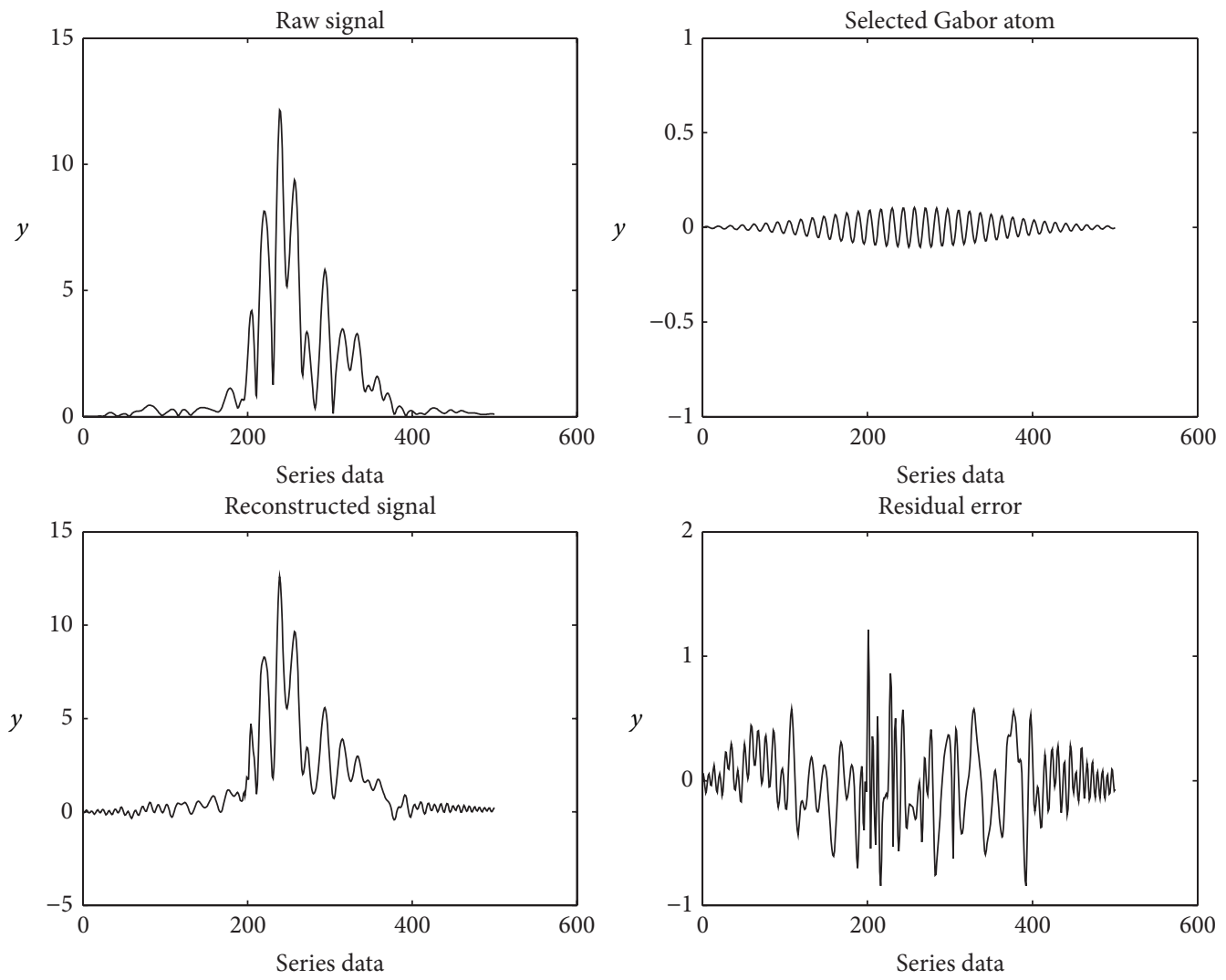

(c)
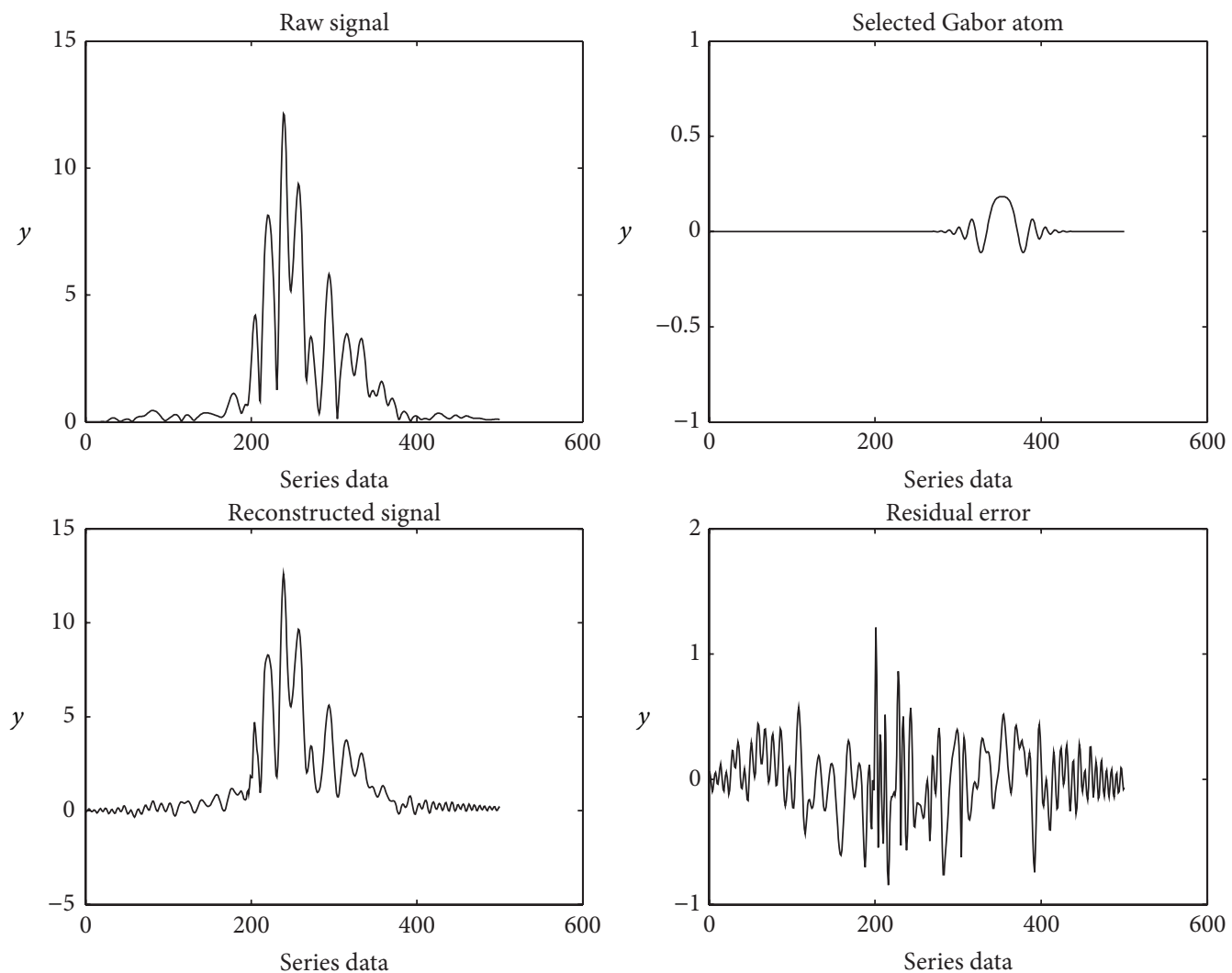

(d)

FIGURE 8: MP decomposition process of falling forward: (a) 1st iteration (b); 2nd iteration; (c) 9th iteration; (d) 10th iteration. 


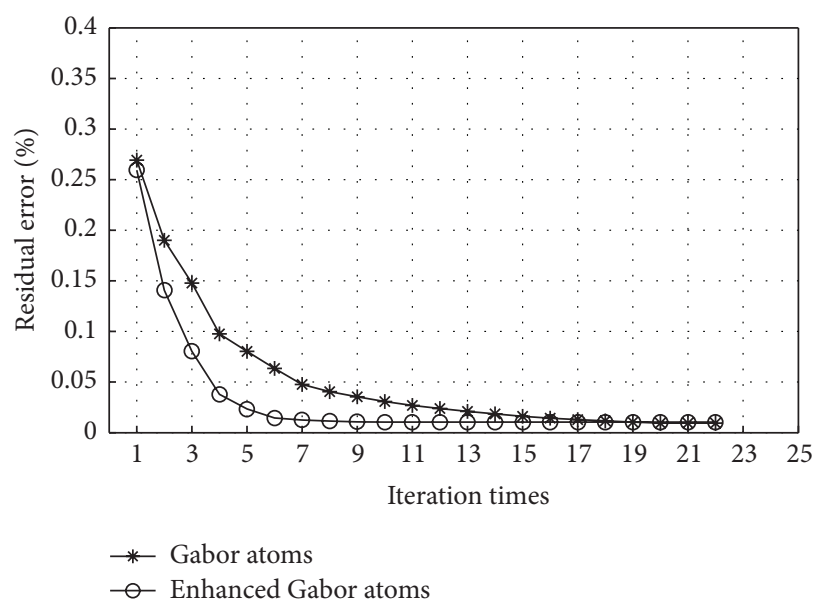

(a)

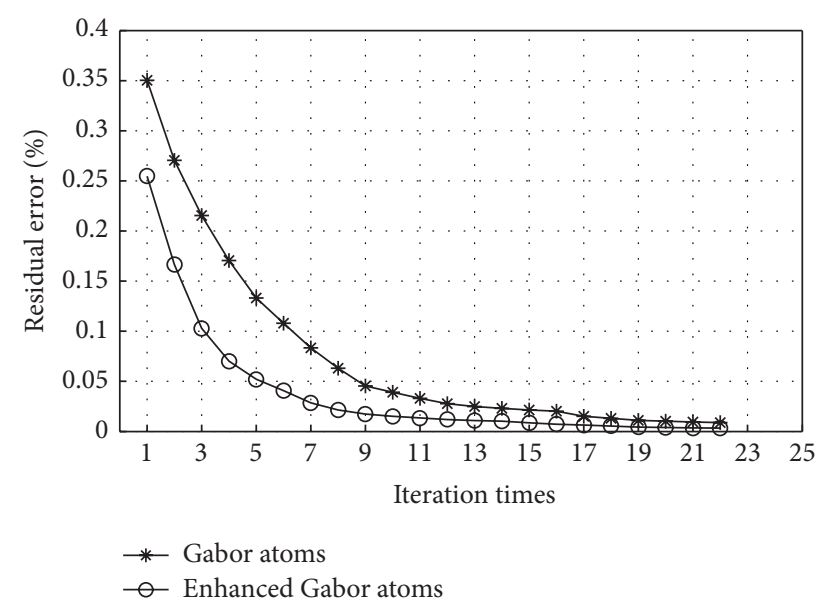

(b)

FIGURE 9: Residual error and the number of iterations in MP process: (a) stumbling; (b) sitting on a chair.

TABLE 1: Parameters of enhanced Gabor atoms after MP decomposition.

\begin{tabular}{ccccc}
\hline & Scale & Time shift & $\begin{array}{c}\text { Frequency } \\
(\mathrm{rad} / \mathrm{s})\end{array}$ & $\begin{array}{c}\text { Phase modulation } \\
\text { rate }\end{array}$ \\
\hline 1 & 128 & 256 & 0 & -0.00019175 \\
2 & 64 & 256 & 6.0377 & 0.0084369 \\
3 & 32 & 240 & 0.19635 & -0.0092039 \\
4 & 256 & 256 & 6.1973 & -0.0042184 \\
5 & 16 & 216 & 0.3927 & -0.061359 \\
6 & 128 & 320 & 6.1605 & 0.0061359 \\
7 & 128 & 192 & 6.2586 & -0.0040267 \\
8 & 16 & 264 & 0.19635 & 0.036816 \\
9 & 256 & 256 & 5.8169 & -0.00014381 \\
10 & 64 & 352 & 6.2832 & 0.0092039 \\
\hline
\end{tabular}

of enhanced Gabor atoms after MP decomposition with ten iterations.

Figure 9 shows the relation between residual error $e_{m}$ and iteration times in MP decomposition process. We set the end condition for iteration as

$$
\begin{aligned}
e_{m}-e_{m-1} & >\varepsilon, \\
e_{m} & =R^{m} x=\left\langle R^{m} x, g_{\gamma_{m}}\right\rangle g_{\gamma_{m}}+R^{m+1} x .
\end{aligned}
$$

Figure 10 shows time-frequent WV distribution of some abnormal behaviors. The WVD owns the highest signal energy concentration in the time-frequency plane for linearly modulated signals, but major problems are the presence of cross-terms for multicomponent signals which can corrupt the transform space [31]. The Gabor-Wigner transform [32] was then introduced to avoid the cross-terms and the noisy character of WVD. In our paper, the enhanced Gabor atom decomposition is preferred by introducing the phase modulation rate in traditional Gabor decomposition. And, from Figure 9, it is clear that our enhanced Gabor atom decomposition has advantages in both iteration times and cost times which own the higher signal energy concentration in time-frequency plane. The discrete WVD energy matrices after enhanced Gabor decomposition are then used for training and recognition by the proposed incremental cloudSVM learning method.

The recognition results are shown in Table 2 . The category and criteria for the evaluation of performance and procedures to carry out the tests refers to [33]. The Activities of Daily Living (ADL) like normal walking, sitting on a chair, climbing steps, and lying on a bed are classified into normal gait behaviors. Other activities in Table 2 belong to abnormal gait behaviors. In the detection processes, it turns out that the binary accuracy recognition rate can be detected nearly $100 \%$ for daily life activities. The results are similar with the method proposed in $[12,17]$. This is because only Booleans of falling down or not as binary results need to be checked out to determine if it is needed to trigger alarm. However, in our daily life, the class of abnormal behaviors and actions must be recognized in order to promote healthy lifestyles for the elderly and chronic patients. In [10-18] a threshold estimated from training sample statistics is used to distinguish fall events. The threshold estimate algorithms can be achieved off-line by computers and the recognition process could be realized in mobile devices with simple algorithms. However, they are sometimes limited in actual applications due to small-scale and static imitated training data.

In order to overcome the limitation of the existing methods, the Wigner-Ville time-frequency analysis method is introduced and the cloud based incremental SVM learning method is used for abnormal behavior detection and classification. The CI-SVM classifier is trained to differentiate between the different action states listed in Table 2 instead of a simple binary classification of falling or not falling. And the Rank-1 recognition rates of abnormal behaviors are listed in Table 2 in three different conditions denoted by $I_{0}, I_{1}$, and $I_{2}$. The recognition rate of $I_{0}$ is calculated by $\Omega_{o}^{0}$ SVM classifier trained using only original 1200 imitated data samples. 

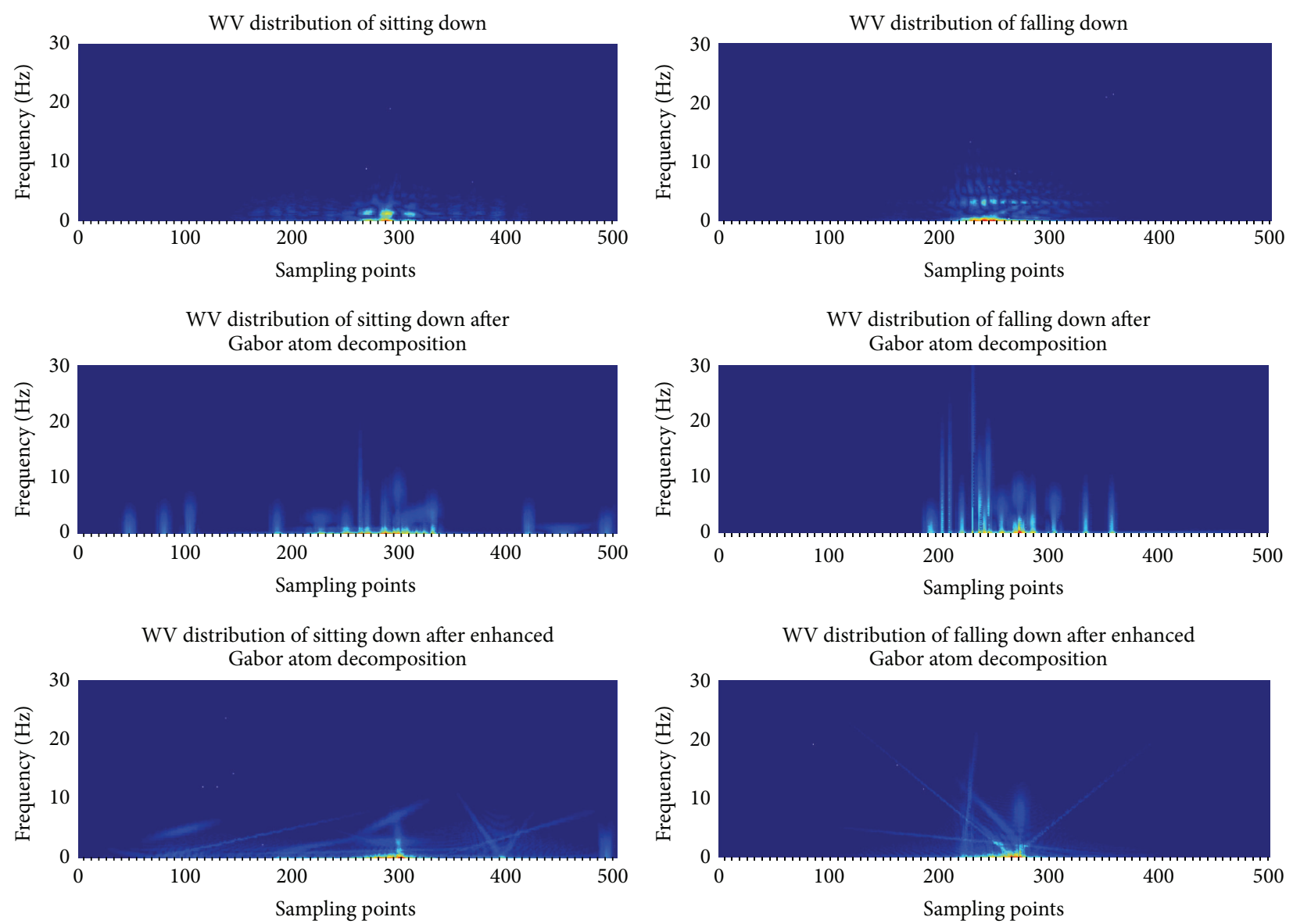

(a)

(b)

FIGURE 10: WV distribution for (a) sitting down on a chair and (b) falling down forward and ending lying.

The mean recognition rates are the lowest of the three, because the actual data of abnormal behaviors collected from 45 to 55 years old peoples are not participated in training. The recognition rate of $I_{1}$ is obtained by the updated $\Omega_{o}^{1}$ SVM classifier after the first cloud based incremental SVM learning. The recognition rates are improved due to the new knowledge accumulated from the sample data of 45 years old peoples. The recognition rate of $I_{2}$ is got by the updated $\Omega_{o}^{2}$ SVM classifier after the second CI-SVM learning. It has the highest rates because the last sample data from 55 years old peoples are participated in incremental learning process. From the experimental data, it can be seen that the imitated sample data in young volunteers cannot fully replace the actual gait and falling of the elderly in training due to the age and physiological differences. The online incremental SVM learning is necessary when the system is put into actual applications.

In Figure 11, cumulative match characteristic (CMC) curve is used to illustrate our abnormal action recognition results compared with other different methods for quantitative analysis. One method is proposed in [12] using machine learning methods based on $K$-nearest neighbor algorithm for human movement classification. The other is discussed in [17] using one-class SVM classification algorithm.
From the results, it can be seen that our method achieves significantly better performance due to the enhanced Wigner-Ville analysis and cloud based incremental SVM Learning. Some existed methods might not have low recognition results when the data are in small scale and the samples are all from the imitated data. It occurs in $I_{0}$ conditions using only original 1200 imitated samples for training and recognition. In such case, our proposed new novel method also has higher recognition rate than the traditional approaches, but not that much as shown in the experimental results. The advantage of our method is in large-scale and actual abnormal behaviors data training and classification by introducing the dynamic online incremental SVM learning method. In real applications, we might not have so many reliable data especially the actual data of elderly to construct training and recognition. And only fixing a threshold to determine the daily activates of human is less reliable and not that robust. Using the time-frequency analysis and the CI-SVM learning methods with an elimination algorithm can improve not only the accuracy of the system but also the cost of time and space that make it possible to run online.

As discussed before, most of the existing methods for elderly abnormal behavior detection are only to check out 


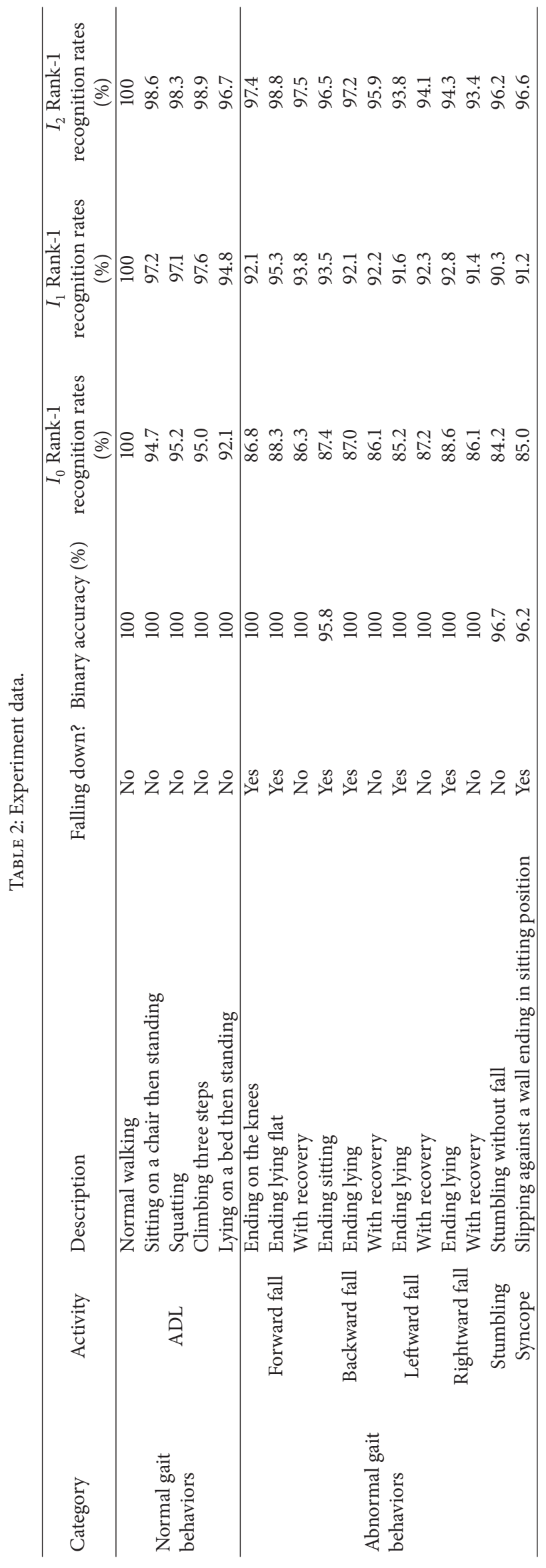




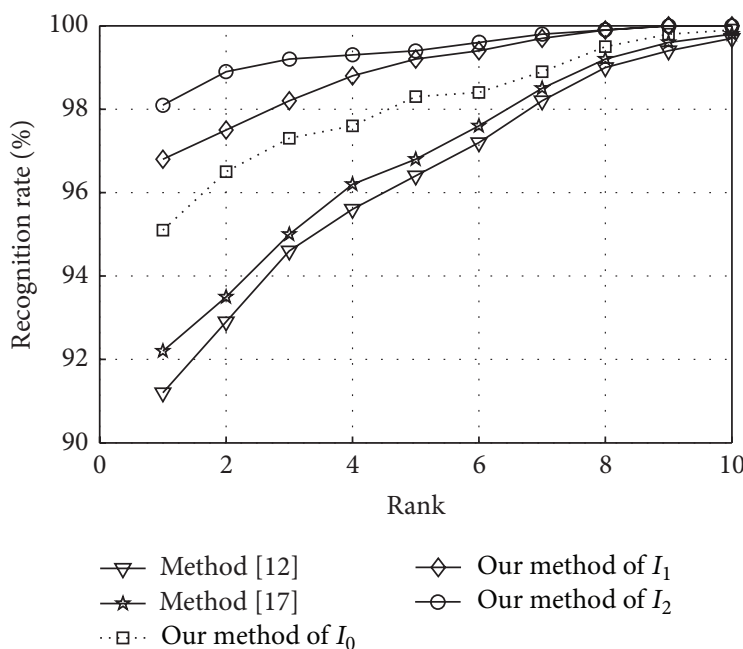

(a)

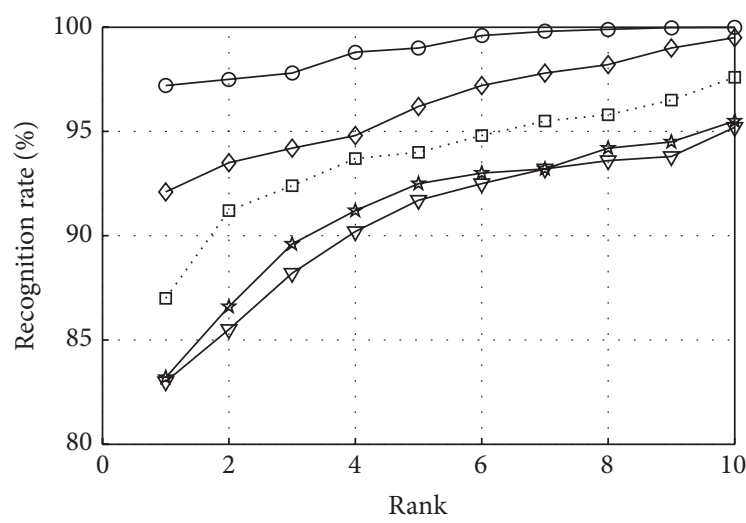

(c)

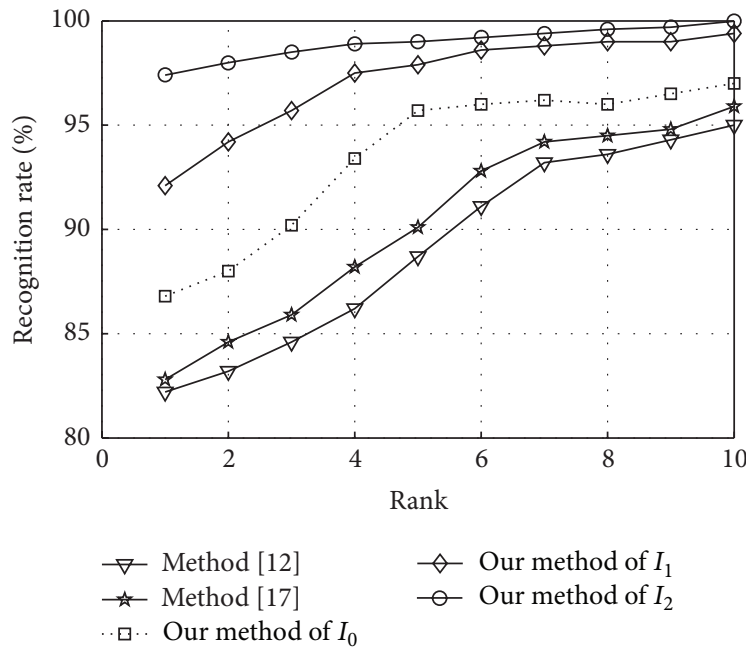

(e)
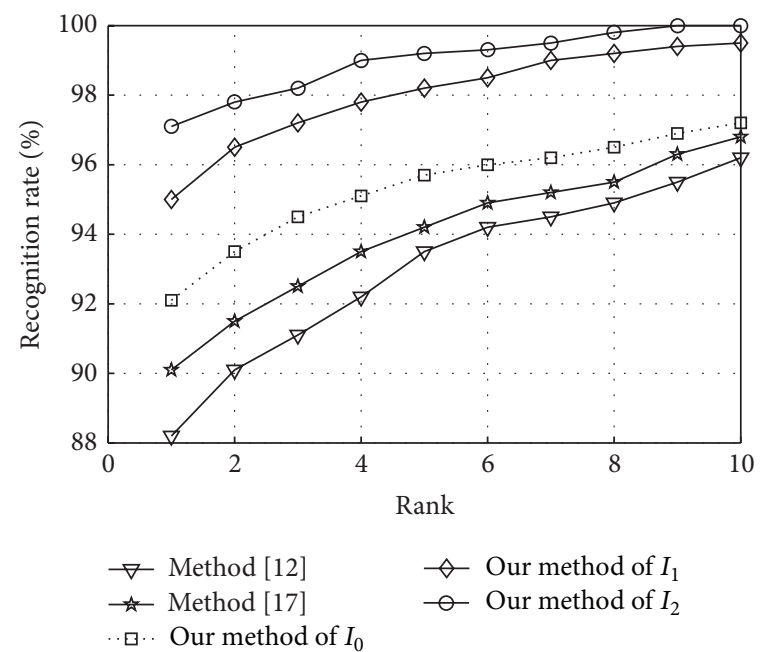

(b)

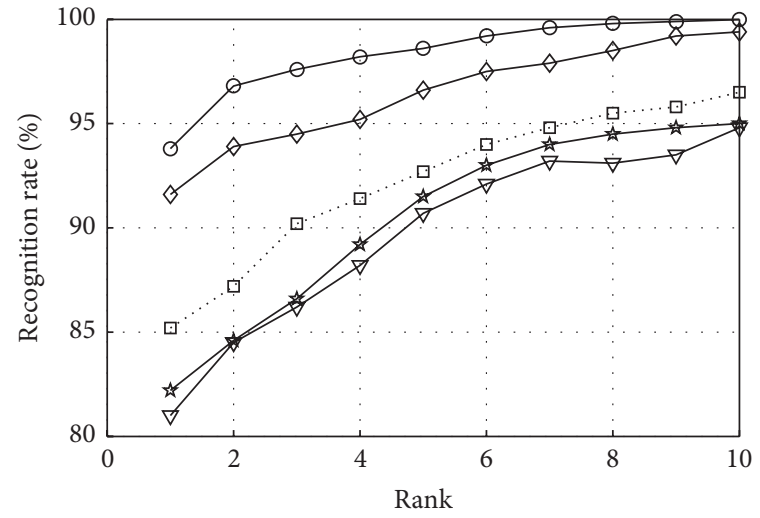

$$
\begin{array}{ll}
\nabla \text { Method [12] } & \smile \text { Our method of } I_{1} \\
- \text { Method [17] } & - \text { Our method of } I_{2} \\
\therefore \text {. } & \text {. Our method of } I_{0}
\end{array}
$$

(d)

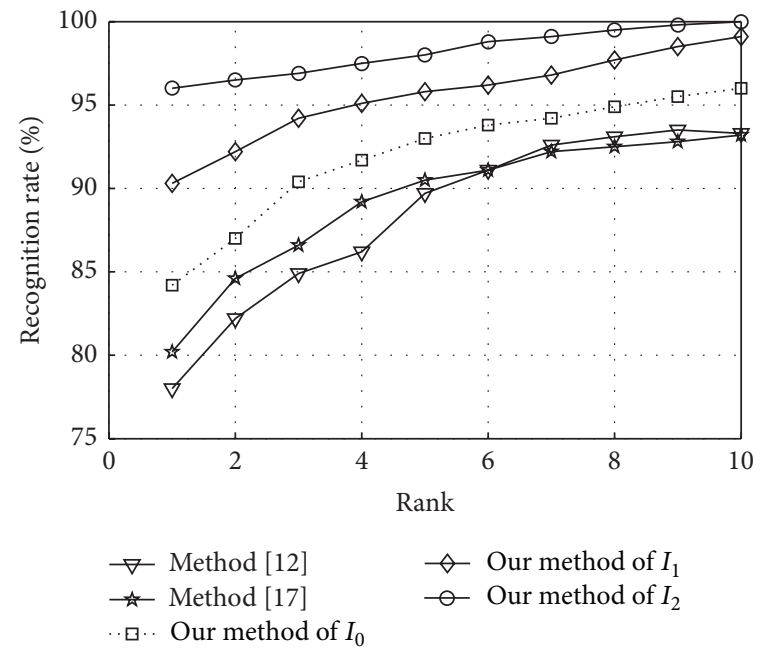

(f)

FIGURE 11: Comparison of recognition results with different methods: (a) squatting; (b) lying on a bed then standing; (c) backward fall ending lying; (d) leftward fall ending lying; (e) forward fall ending on the knees; (f) stumbling without fall. 
TABLE 3: Comparison results of different methods for activities classification.

\begin{tabular}{|c|c|c|c|c|c|c|}
\hline Experiment & BPNN [12] & Method [34] & Method [35] & Method [36] & Method [37] & Our method \\
\hline Walking & $95.0 \%$ & $92.2 \%$ & $90.0 \%$ & $99.9 \%$ & $94.2 \%$ & $100 \%$ \\
\hline Sitting & $91.6 \%$ & $90.2 \%$ & $96.7 \%$ & $90.6 \%$ & $91.7 \%$ & $98.6 \%$ \\
\hline Lying & $90.0 \%$ & $98.4 \%$ & $74.1 \%$ & $83.3 \%$ & - & $96.7 \%$ \\
\hline Falling & $86.6 \%$ & - & $95.5 \%$ & $87.5 \%$ & - & $95.9 \%$ \\
\hline Squatting & - & - & - & - & - & $98.3 \%$ \\
\hline Stumbling & - & - & - & - & - & $96.2 \%$ \\
\hline Syncope & - & - & - & - & - & $96.6 \%$ \\
\hline Average & $90.8 \%$ & $93.6 \%$ & $89.1 \%$ & $90.3 \%$ & $92.9 \%$ & $97.4 \%$ \\
\hline
\end{tabular}

whether the object is falling or not. It is only a 2-class problem and the output is a binary one. However, performing only binary realization for elderly care system is insufficient. In order to solve the problem, this paper proposed an abnormal behavior detection system for elderly health care based on CI-SVM classifier to recognize the human behaviors and actions. Some methods [34-37] have been proposed for human activity classification. However, most of them are not aimed at the problem of elderly care. As a result the classifications of the activities are limited to few categories, for example, walking, sitting, falling, and lying. In [34] a method of physical activity monitoring is presented, which is able to detect body postures and periods of walking using one kinematic sensor attached to the chest. However, only three postures, including sitting, standing, and lying, are collected for classification. In [35], it presents the implementation of a realtime classification system for the types of human movement with waist-mounted triaxial accelerometer unit. The system uses multiple threshold and sum of the values (as opposed to their mean) for the comparison and classification. In [36] neural classifier is introduced for human activities classifying using a triaxial accelerometer. However, the classifier could be improved to recognize more complex activities accurately. In [37] a human activity classifier is presented, based on the real-time analysis of the signals detected with a triaxial accelerometer fixed to the trunk. Two thresholds were defined based on a database of stereotyped activities for classification.

Differing from the existing methods with limited categories of human activities, in this paper, the subclasses of the abnormal activities for elderly care are introduced for classification, for example, falling with different conditions. Some special situations like stumbling and syncope are added for classification. In Table 3, some classification methods for activities are compared and results of the experiments that are not presented in the original papers have been left blank in the table. Our results shown in Table 3 are the mean value calculated from Table 2. The existing methods show high recognition results when the activities are normal walking or sitting. However, the classification for behaviors like lying and falling has low recognition rate. This is because the threshold of the normal lying and abnormal falling are much the same. If the method is based on threshold for classification, these two activities might be mismatched. In contrast, the performance of our time-frequency analysis and CI-SVM based algorithm shows satisfactory classification results. In method [34], the lying recognition is about $98.4 \%$. It is because only three postures are classified without the falling detection. And the falling recognition rate in method [35] is $95.5 \%$ high. It is because only 15 samples are tested for falling detection, while about 1000 samples falling data were used in our experiments. The dataset used in our experiments are much larger than the methods compared in Table 3. However, our approach outperforms all the existing methods. There are several reasons why our method achieves significantly better performance. One is the time-frequency analysis method used in our algorithm. It provided easier extraction of the specific motion features and captures the instantaneous frequency laws that are most suitable for the underlying application. And preprocess of the strengthen Kalman filter and enhanced Gabor atoms decomposition also helps to enhanced the useful features while mitigate the useless noise signals. The other reason is introducing the cloud based incremental learning method into the health care system. Compared with the traditional methods like KNN, SVM, and so forth, the CI-SVM helps to construct the more accuracy classifier for abnormal behavior detection.

\section{Conclusion}

In this paper, three-axis acceleration sensors embedded in the intelligent mobile terminal are used to collect, preprocess, and transmit the data of abnormal behaviors for the elderly. The motion features of abnormal gait behaviors are processed and extracted by enhanced Wigner-Ville time-frequency analysis method. A cloud based incremental SVM (CI-SVM) learning method is proposed for abnormal behavior classification and recognition. By using the CI-SVM, the knowledge is accumulated in the process of cloud based incremental learning, which helps to construct the more accuracy classifier for abnormal behavior detection. As a result, the abnormal behaviors can be incrementally trained and distinguished online. The results are pushed to a Remote Telecare Center and clients are associated with the topics in real-time. The proposed method is feasible and it can be applied to the care for the age, emergency aid, and related fields.

\section{Conflict of Interests}

The authors declare no conflict of interests. 


\section{Authors' Contribution}

All authors participated in designing and implementing the recognition method. All authors discussed the basic structure of the paper. Jian Luo drafted the main parts of the paper. Jin Tang and Xiaoming Xiao reviewed and edited the draft. All authors read and approved the final paper.

\section{Acknowledgments}

This work was supported partly by the National Natural Science Foundation of China (Grants no. 61403426 and no. 91220301), the Scientific Research Project of Hunan Provincial Education Department, China (Grant no. 15C0981), and the Science and Technology Key Program of Hunan, China (Grant no. 2015WK3006).

\section{References}

[1] United Nations, Department of Economic and Social Affairs, and Population Division, World Population Ageing 2013, United Nations Publications, New York, NY, USA, 2013.

[2] M. J. Gibson, R. O. Andres, T. E. Kennedy et al., "The prevention of falls in later life. A report of the Kellogg International Work Group on the Prevention of Falls by the Elderly," Danish Medical Bulletin, vol. 34, supplement 4, pp. 1-24, 1987.

[3] L. Palmerini, F. Bagalà, A. Zanetti, J. Klenk, C. Becker, and A. Cappello, "A wavelet-based approach to fall detection," Sensors, vol. 15, no. 5, pp. 11575-11586, 2015.

[4] T. Xiang and S. Gong, "Incremental and adaptive abnormal behaviour detection," Computer Vision and Image Understanding, vol. 111, no. 1, pp. 59-73, 2008.

[5] D. Zhang, H. Peng, Y. Haibin, and Y. Lu, "Crowd abnormal behavior detection based on machine learning," Information Technology Journal, vol. 12, no. 6, pp. 1199-1205, 2013.

[6] C. Bauckhage, J. K. Tsotsos, and F. E. Bunn, "Automatic detection of abnormal gait," Image and Vision Computing, vol. 27, no. 1-2, pp. 108-115, 2009.

[7] Z. A. Khan and W. Sohn, "Abnormal human activity recognition system based on R-transform and kernel discriminant technique for elderly home care," IEEE Transactions on Consumer Electronics, vol. 57, no. 4, pp. 1843-1850, 2011.

[8] X. Ma, H. Wang, B. Xue, M. Zhou, B. Ji, and Y. Li, "Depthbased human fall detection via shape features and improved extreme learning machine," IEEE Journal of Biomedical and Health Informatics, vol. 18, no. 6, pp. 1915-1922, 2014.

[9] J. Tang, J. Luo, T. Tjahjadi, and Y. Gao, "2.5D multi-view gait recognition based on point cloud registration," Sensors (Switzerland), vol. 14, no. 4, pp. 6124-6143, 2014.

[10] Q. T. Huynh, U. D. Nguyen, L. B. Irazabal, N. Ghassemian, and B. Q. Tran, "Optimization of an accelerometer and gyroscopebased fall detection algorithm," Journal of Sensors, vol. 2015, Article ID 452078, 8 pages, 2015.

[11] B. Pogorelc, Z. Bosnić, and M. Gams, "Automatic recognition of gait-related health problems in the elderly using machine learning," Multimedia Tools and Applications, vol. 58, no. 2, pp. 333-354, 2012.

[12] C. Li, M. Lin, L. T. Yang, and C. Ding, "Integrating the enriched feature with machine learning algorithms for human movement and fall detection," Journal of Supercomputing, vol. 67, no. 3, pp. 854-865, 2014.
[13] D. Naranjo-Hernández, L. M. Roa, J. Reina-Tosina, and M. Á. Estudillo-Valderrama, "SoM: a smart sensor for human activity monitoring and assisted healthy ageing," IEEE Transactions on Biomedical Engineering, vol. 59, no. 12, pp. 3177-3184, 2012.

[14] M. N. Nyan, F. E. H. Tay, and E. Murugasu, "A wearable system for pre-impact fall detection," Journal of Biomechanics, vol. 41, no. 16, pp. 3475-3481, 2008.

[15] A. K. Bourke and G. M. Lyons, "A threshold-based falldetection algorithm using a bi-axial gyroscope sensor," Medical Engineering and Physics, vol. 30, no. 1, pp. 84-90, 2008.

[16] A. K. Bourke, K. J. O’Donovan, and G. ÓLaighin, “The identification of vertical velocity profiles using an inertial sensor to investigate pre-impact detection of falls," Medical Engineering and Physics, vol. 30, no. 7, pp. 937-946, 2008.

[17] Z. Ye, Y. Li, Q. Zhao, and X. Liu, "A falling detection system with wireless sensor for the elderly people based on ergnomics," International Journal of Smart Home, vol. 8, no. 1, pp. 187-196, 2014.

[18] D. Curone, G. M. Bertolotti, A. Cristiani, E. L. Secco, and G. Magenes, "A real-time and self-calibrating algorithm based on triaxial accelerometer signals for the detection of human posture and activity," IEEE Transactions on Information Technology in Biomedicine, vol. 14, no. 4, pp. 1098-1105, 2010.

[19] I. Orović, S. Stanković, and M. Amin, "A new approach for classification of human gait based on time-frequency feature representations," Signal Processing, vol. 91, no. 6, pp. 1448-1456, 2011.

[20] E. G. Davis, A. Calveras, and I. Demirkol, "Improving packet delivery performance of publish/subscribe protocols in wireless sensor networks," Sensors, vol. 13, no. 1, pp. 648-680, 2013.

[21] N. Ma, M. Bouchard, and R. A. Goubran, "Speech enhancement using a masking threshold constrained kalman filter and its heuristic implementations," IEEE Transactions on Audio, Speech and Language Processing, vol. 14, no. 1, pp. 19-31, 2006.

[22] S. Gannot, "Speech processing utilizing the Kalman filter," IEEE Instrumentation \& Measurement Magazine, vol. 15, no. 3, pp. 10$14,2012$.

[23] B. Trzaskowski, W. W. Jedrzejczak, E. Pilka, K. Kochanek, and H. Skarzynski, "Automatic removal of sonomotor waves from auditory brainstem responses," Computers in Biology and Medicine, vol. 43, no. 5, pp. 524-532, 2013.

[24] R. Gribonval, "Fast matching pursuit with a multiscale dictionary of Gaussian chirps," IEEE Transactions on Signal Processing, vol. 49, no. 5, pp. 994-1001, 2001.

[25] R. Kuś, P. T. Różański, and P. J. Durka, "Multivariate matching pursuit in optimal Gabor dictionaries: theory and software with interface for EEG/MEG via Svarog," BioMedical Engineering OnLine, vol. 12, article 94, 2013.

[26] V. N. Vapnik, The Nature of Statistical Learning Theory, Springer, New York, NY, USA, 1998.

[27] J. Zheng, F. Shen, H. Fan, and J. Zhao, "An online incremental learning support vector machine for large-scale data," Neural Computing and Applications, vol. 22, no. 5, pp. 1023-1035, 2013.

[28] R. Chitrakar and C. Huang, "Selection of Candidate Support Vectors in incremental SVM for network intrusion detection," Computers \& Security, vol. 45, pp. 231-241, 2014.

[29] W. Wang, "An incremental learning strategy for support vector regression," Neural Processing Letters, vol. 21, no. 3, pp. 175-188, 2005.

[30] R. Xiao, J. Wang, and F. Zhang, "An approach to incremental SVM learning algorithm," in Proceedings of the 12th IEEE 
International Conference on Tools with Artificial Intelligence (ICTAI '00), pp. 268-273, IEEE, Vancouver, Canada, November 2000.

[31] R. B. Pachori and P. Sircar, "A new technique to reduce cross terms in the Wigner distribution," Digital Signal Processing, vol. 17, no. 2, pp. 466-474, 2007.

[32] S.-C. Pei and J.-J. Ding, "Relations between gabor transforms and fractional fourier transforms and their applications for signal processing," IEEE Transactions on Signal Processing, vol. 55, no. 10, pp. 4839-4850, 2007.

[33] P. Paola, B. Alberto, P. Lorenzo, P. Marco, P. Luca, and V. Simone, "A high reliability wearable device for elderly fall detection," IEEE Sensors Journal, vol. 15, pp. 4544-4553, 2015.

[34] B. Najafi, K. Aminian, A. Paraschiv-Ionescu, F. Loew, C. J. Büla, and P. Robert, "Ambulatory system for human motion analysis using a kinematic sensor: monitoring of daily physical activity in the elderly," IEEE Transactions on Biomedical Engineering, vol. 50, no. 6, pp. 711-723, 2003.

[35] D. M. Karantonis, M. R. Narayanan, M. Mathie, N. H. Lovell, and B. G. Celler, "Implementation of a real-time human movement classifier using a triaxial accelerometer for ambulatory monitoring," IEEE Transactions on Information Technology in Biomedicine, vol. 10, no. 1, pp. 156-167, 2006.

[36] J.-Y. Yang, J.-S. Wang, and Y.-P. Chen, "Using acceleration measurements for activity recognition: an effective learning algorithm for constructing neural classifiers," Pattern Recognition Letters, vol. 29, no. 16, pp. 2213-2220, 2008.

[37] D. Curone, G. M. Bertolotti, A. Cristiani, E. L. Secco, and G. Magenes, "A real-time and self-calibrating algorithm based on triaxial accelerometer signals for the detection of human posture and activity," IEEE Transactions on Information Technology in Biomedicine, vol. 14, no. 4, pp. 1098-1105, 2010. 


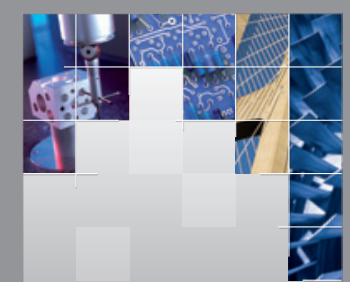

\section{Enfincering}
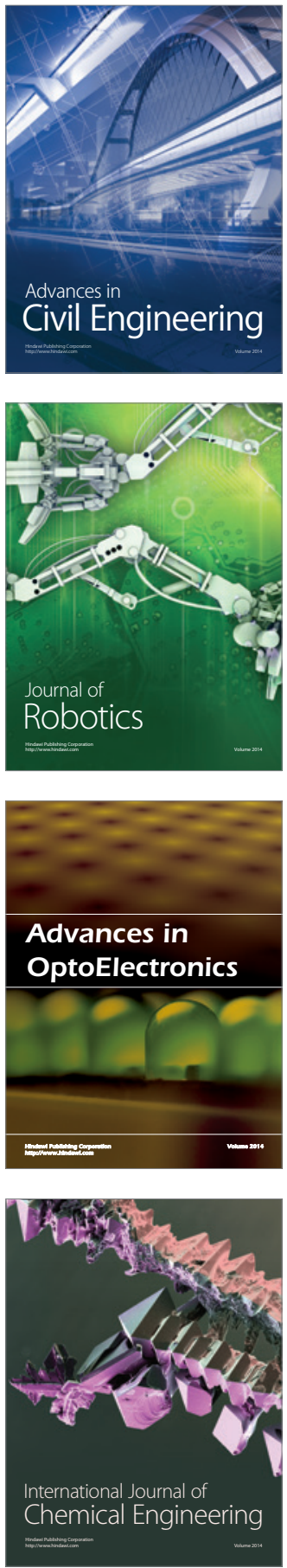

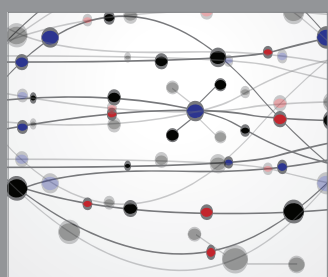

The Scientific World Journal

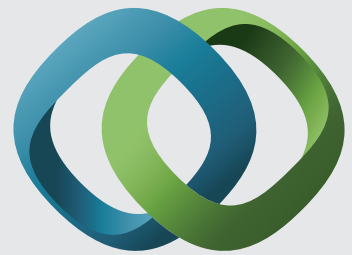

\section{Hindawi}

Submit your manuscripts at

http://www.hindawi.com
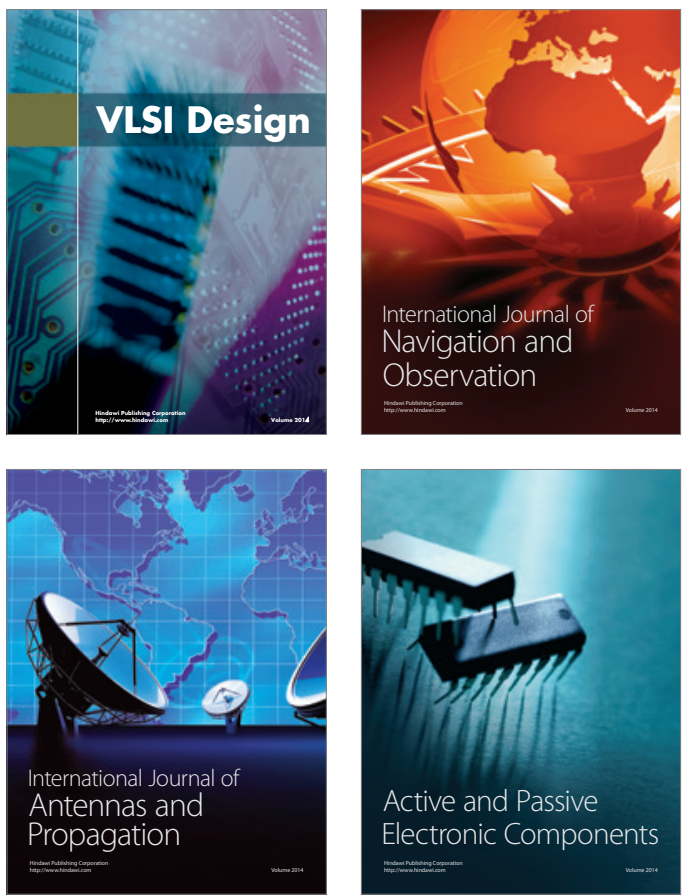
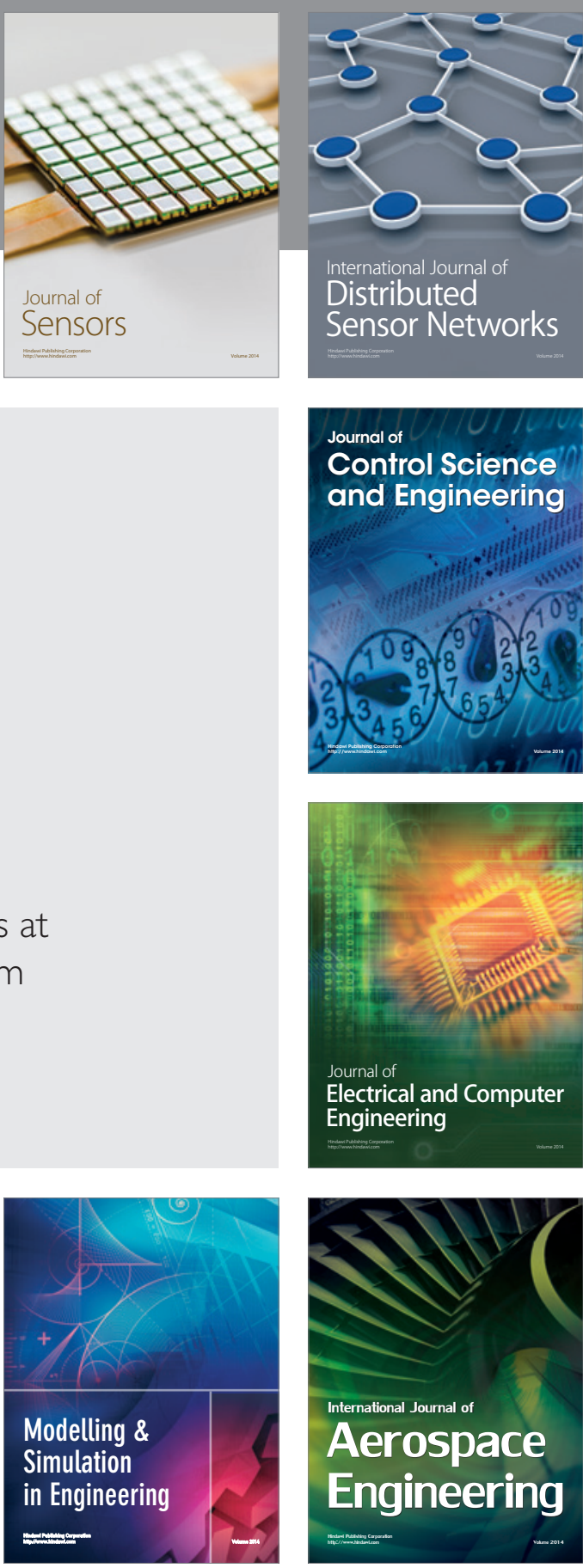

International Journal of

Distributed

Sensor Networks

Journal of

Control Science

and Engineering
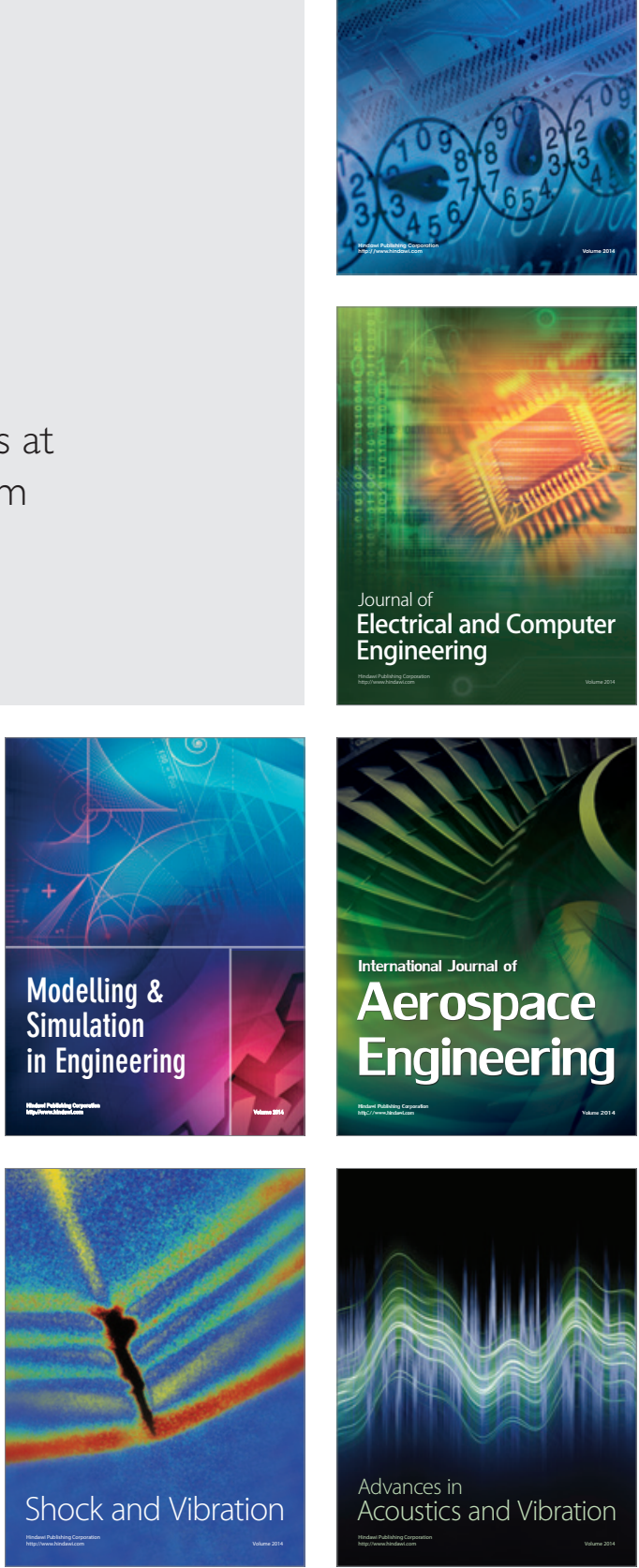